\title{
Low-energy effective action in two-dimensional SQED: a two-loop analysis
}

\author{
I.B. Samsonov \\ Bogoliubov Laboratory of Theoretical Physics, JINR, \\ 141980 Dubna, Moscow region, Russia \\ E-mail: samsonov@theor.jinr.ru
}

ABSTRACT: We study two-loop quantum corrections to the low-energy effective actions in $\mathcal{N}=(2,2)$ and $\mathcal{N}=(4,4)$ SQED on the Coulomb branch. In the latter model, the low-energy effective action is described by a generalized Kähler potential which depends on both chiral and twisted chiral superfields. We demonstrate that this generalized Kähler potential is one-loop exact and corresponds to the $\mathcal{N}=(4,4)$ sigma-model with torsion presented by Roček, Schoutens and Sevrin [1]. In the $\mathcal{N}=(2,2) \mathrm{SQED}$, the effective Kähler potential is not protected against higher-loop quantum corrections. The two-loop quantum corrections to this potential and the corresponding sigma-model metric are explicitly found.

KEYwords: Extended Supersymmetry, Supersymmetric Gauge Theory, Field Theories in Lower Dimensions, Superspaces

ARXIV EPRINT: 1704.04148 


\section{Contents}

1 Introduction and summary 1

2 Exact propagators on constant vector multiplet background 4

2.1 Gauge theory in $\mathcal{N}=(2,2)$ superspace 4

2.2 Parallel displacement propagator in $\mathcal{N}=(2,2)$ superspace 6

2.3 Real superfield Green's function and its heat kernel 8

$\begin{array}{lr}2.4 \text { Heat kernel for chiral superfield Green's function } & 10\end{array}$

2.5 Heat kernel for Green's function $G_{+-} \quad 12$

3 Low-energy effective action in $\mathcal{N}=(2,2)$ SQED 13

$\begin{array}{lll}3.1 & \text { General remarks } & 13\end{array}$

$\begin{array}{ll}\text { 3.2 SQED with one chiral flavor } & 15\end{array}$

$\begin{array}{lll}3.2 .1 & \text { Classical action } & 15\end{array}$

$\begin{array}{ll}\text { 3.2.2 Even part of the one-loop effective action } & 16\end{array}$

$\begin{array}{ll}3.2 .3 \text { Odd part of the one-loop effective action } & 18\end{array}$

$\begin{array}{lll}\text { 3.3 SQED with two chiral flavors } & 20\end{array}$

$\begin{array}{lll}\text { 3.3.1 Classical action and background field setup } & 20\end{array}$

$\begin{array}{ll}\text { 3.3.2 One-loop effective action } & 22\end{array}$

3.3.3 Two-loop effective Kähler potential 24

4 Low-energy effective action in $\mathcal{N}=(4,4)$ SQED 28

4.1 Classical action and loop expansion of the effective action 28

4.2 One-loop effective action and the Wess-Zumino term 30

4.3 Vanishing of two-loop corrections to generalized Kähler potential 32

$\begin{array}{llr}5 & \text { Conclusions } & 34\end{array}$

A $\mathcal{N}=(2,2)$ superspace conventions $\quad 35$

\section{Introduction and summary}

Two-dimensional supersymmetric gauge theories have a wide range of applications in physics and geometry. In field theory, $2 \mathrm{~d}$ gauged linear sigma-models in $\mathcal{N}=(2,2)$ superspace serve as canonical examples which provide very useful insights on low-energy dynamics of four-dimensional supersymmetric gauge theories $[2,3]$. Geometrically, twodimensional non-linear sigma-models with extended supersymmetry appear very reach because of existence of numerous twisted-chiral multiplets [4] which possess no analogs in higher dimensions. In particular, such sigma-models may support Kähler, hyper-Kähler or 
generalized Kähler geometry. It is natural to expect that some of these geometries may arise as low-energy effective actions in two-dimensional gauge theories in $\mathcal{N}=(2,2)$ superspace.

The study of low-energy effective action in Abelian gauge theories in $\mathcal{N}=(2,2)$ superspace was initiated long ago [2]. The authors of this work showed that the field strength of $\mathcal{N}=(2,2)$ vector multiplet is given by a twisted chiral superfield which we denote by $\Sigma$ throughout this work. The effective action for $\Sigma$ may have a superpotential $W(\Sigma)$ and a Kähler potential $K(\Sigma, \bar{\Sigma})$, as well as higher-derivative terms which form together the Euler-Heisenberg-type effective action in two-dimensional SQED. The structure of one-loop quantum corrections to these potentials was found in [2]:

$$
\begin{aligned}
W^{(1)}(\Sigma) & \propto \Sigma \ln \Sigma-\Sigma, \\
K^{(1)}(\Sigma, \bar{\Sigma}) & \propto \ln \Sigma \ln \bar{\Sigma} .
\end{aligned}
$$

At leading order, one can discard higher-derivative terms in the effective action and treat the low-energy theory as a $(2,2)$ sigma-model with the Kähler potential (1.2) and superpotential (1.1).

The superpotential (1.1) is known to be one-loop exact and its form is completely determined by the anomaly of $\mathrm{U}(1) \times \mathrm{U}(1)$ R-symmetry [2]. The Kähler potential can, however, receive higher-loop quantum corrections. This paper aims to trigger the study of quantum corrections to the effective Kähler potential $K(\Sigma, \bar{\Sigma})$ and corresponding sigmamodel geometry beyond one-loop order.

We consider two-loop effective action in $\mathcal{N}=(2,2)$ and $(4,4)$ SQED on the Coulomb branch. In the $(2,2)$ case, the Coulomb branch is known to exist only when the $\mathrm{U}(1)$ charges of chiral multiplets sum to zero $[3,5]$. This is typically satisfied for the SQED with two chiral multiplets which carry opposite charges with respect to the gauge group. For this theory we explicitly compute two-loop quantum corrections to the effective Kähler potential $K^{(2)}(\Sigma, \bar{\Sigma})$.

An important feature of two-dimensional gauge theories is that Feynman graphs with internal (super)photon lines suffer from IR divergencies. We show that for supersymmetric gauge theories in the $\mathcal{N}=(2,2)$ superspace it is possible to introduce gauge invariant mass term for the vector multiplet which naturally regulates such IR divergencies. This mass term may be obtained by the dimensional reduction from the three-dimensional (super) Chern-Simons action which is also known to be responsible for the gauge-invariant mass of the vector multiplet in three dimensions. In our case, the two-loop quantum corrections to the effective action explicitly depend on the vector multiplet mass and are singular in the limit when this mass vanishes.

The $(4,4)$ vector multiplet in the $\mathcal{N}=(2,2)$ superspace is described by the pair $(\Phi, \Sigma)$ where $\Phi$ is a chiral and $\Sigma$ is a twisted chiral multiplet [4]. At leading order in the derivative expansion, the low-energy effective action in the $\mathcal{N}=(4,4)$ SQED is described by a generalized Kähler potential $K(\Sigma, \bar{\Sigma} ; \Phi, \bar{\Phi})$. Performing explicit quantum computations we demonstrate that this potential does not receive two-loop quantum corrections and is one-loop exact. At one-loop order, this function coincides with the potential for the $(4,4)$ 
sigma-model with torsion studied in $[1]^{1}$

$$
K^{(1)}(\Sigma, \bar{\Sigma} ; \Phi, \bar{\Phi})=\frac{1}{4 \pi}\left[\ln \Sigma \ln \bar{\Sigma}+\operatorname{Li}_{2}\left(-\frac{\Phi \bar{\Phi}}{\Sigma \bar{\Sigma}}\right)\right]
$$

This sigma-model is known to contain the Wess-Zumino term which has rigid form because of its topological nature $[4,8]$. The coefficient in front of the Wess-Zumino term is oneloop exact and quantizes (see, e.g., [9]). This confirms the non-renormalization of the potential (1.3) claimed in [10].

Qualitatively, the presence of the Wess-Zumino term in the low-energy effective action of $\mathcal{N}=(4,4) \mathrm{SQED}$ is well understood. Indeed, this theory possesses $\mathrm{SO}(4) \simeq \mathrm{SU}(2) \times$ $\mathrm{SU}(2)$ R-symmetry which suffers from the 't Hooft anomaly. At low energy, we integrate out the massive chiral multiplets and consider effective action for the light vector multiplet. However, the total contribution to the anomaly should be the same at low and high energies since the anomaly cannot depend on the energy scale. Thus, the low-energy effective action must include the Wess-Zumino term compensating the contribution to the anomaly from the fermions that were integrated out. This statement is well known as the 't Hooft anomaly matching argument [11].

It is pertinent to mention here the amazing analogy of the effective potentials (1.1), (1.2) and (1.3) with certain terms in low-energy effective actions of fourdimensional $\mathcal{N}=2$ and $\mathcal{N}=4$ gauge theories. Recall that the $4 \mathrm{~d} \mathcal{N}=2$ gauge multiplet may be described by an $\mathcal{N}=2$ chiral superfield $\mathcal{W}$. The superpotential (1.1) is somewhat similar to the so-called holomorphic potential [12] $\mathcal{F}(\mathcal{W}) \propto \mathcal{W}^{2} \ln \mathcal{W}$ while the Kähler potential (1.2) formally coincides with the non-holomorphic potential [13] $\mathcal{H}(\mathcal{W}, \overline{\mathcal{W}}) \propto \ln \mathcal{W} \ln \mathcal{\mathcal { W }}$. This analogy is not accidental: both $\mathcal{F}(\mathcal{W})$ and the superpotential (1.1) appear as a result of integration of the anomaly of U(1) R-symmetry (see [12] and [2], correspondingly). Surprisingly, the potential (1.3) nicely correlates with the lowenergy effective action of $4 \mathrm{~d} \mathcal{N}=4 \mathrm{SYM}$ effective action in the $\mathcal{N}=2$ superspace [14]. Indeed, the first term in the right-hand side of (1.3) formally coincides with the nonholomorphic potential $\mathcal{H}(\mathcal{W}, \overline{\mathcal{W}})$ while the last term in (1.3) is very similar to the hypermultiplet completion of the non-holomorphic potential that was constructed in [14].

This analogy is even more striking. Indeed, in [15] it was demonstrated that the lowenergy effective action in $\mathcal{N}=4 \mathrm{SYM}$ theory contains the Wess-Zumino term for scalar fields which originates from the 't Hooft anomaly matching for the R-symmetry. This Wess-Zumino term implies the non-renormalization of the coefficient in front of the nonholomorphic potential beyond one loop. As we show in this paper, the potential (1.3) is also responsible for the Wess-Zumino term for two-dimensional scalars, and exactly the same arguments provide its non-renormalization.

One of the results of this paper is the illustration of the deep interplay between the two-dimensional $\mathcal{N}=(4,4)$ SQED and $4 \mathrm{~d} \mathcal{N}=4$ SYM theory at low energies, although they are very different in general.

\footnotetext{
${ }^{1}$ This sigma-model can be considered as a particular case of the $\mathcal{N}=(4,4)$ super-Liouville theory which was constructed originally in [6, 7]. I am grateful to E.A. Ivanov for drawing my attention to these works.
} 
This paper is organized as follows. We start the main part of the text (section 2) with a short review of the gauge theory in $\mathcal{N}=(2,2)$ superspace and consider basic properties of the parallel displacement propagator which is a key ingredient of the technique of gauge-covariant perturbative computations (for $4 \mathrm{~d}$ gauge theories in $\mathcal{N}=1$ superspace this technique was developed in [16-18] and for field theory on the supergravity background in [19-22]). Making use of this propagator, we construct exact Green's functions for chiral superfields on covariantly constant vector multiplet background. In section 3 , we compute the low-energy effective action in $\mathcal{N}=(2,2)$ SQED with different numbers of chiral multiplets. We start with a review of old results [2] of one-loop quantum contributions to the effective action and show how they can be naturally reproduced by taking advantage of the technique of covariant perturbative computations in the $\mathcal{N}=(2,2)$ superspace. This technique is then applied to compute two-loop quantum corrections to the effective action of $\mathcal{N}=(2,2)$ SQED on the Coulomb branch. In section 4, we study the structure of low-energy effective action in $\mathcal{N}=(4,4)$ SQED to the two-loop order in perturbation theory and discuss its interplay with the $4 \mathrm{~d} \mathcal{N}=2$ and $\mathcal{N}=4 \mathrm{SYM}$ effective actions. The Conclusions section is devoted to discussions of possible extensions of the results of this work. In appendix we give a summary of our superspace conventions.

\section{Exact propagators on constant vector multiplet background}

In this section we consider two-dimensional non-Abelian gauge theory in $\mathcal{N}=(2,2)$ superspace and, following [18], we introduce parallel displacement propagator which is a key ingredient of gauge-covariant technique of multi-loop quantum computations. Using the properties of this propagator we construct exact heat kernels for basic Green's functions on covariantly constant vector multiplet background. In the Abelian case, we apply these heat kernels in the subsequent sections to compute two-loop quantum correction to the effective action. We hope that the results of this section will be of use also for the study of effective action in non-Abelian gauge theories which will be considered elsewhere. We keep the structure of this section close to the corresponding presentation in [23] to facilitate the comparison with the three-dimensional gauge theory in $\mathcal{N}=2$ superspace.

\subsection{Gauge theory in $\mathcal{N}=(2,2)$ superspace}

We consider the two-dimensional $\mathcal{N}=(2,2)$ superspace with coordinates $z^{A}=\left(x^{m}, \theta^{\alpha}, \bar{\theta}_{\alpha}\right)$, where $x^{m}, m=0,1$, are the Minkowski space coordinates, $\theta^{\alpha}, \alpha=1,2$, are Grassmann coordinates and $\bar{\theta}_{\alpha}=\left(\theta_{\alpha}\right)^{*}$ are their complex conjugate. Our superspace conventions are summarized in appendix. They are chosen to be close to the ones employed in the series of papers [23-27] devoted to the study of superfield theories in three-dimensional $\mathcal{N}=2$ superspace.

The (non-Abelian) gauge theory in the $\mathcal{N}=(2,2)$ superspace is described by the set of gauge-covariant superspace derivatives

$$
\nabla_{A}=\left(\nabla_{m}, \nabla_{\alpha}, \bar{\nabla}^{\alpha}\right)=D_{A}+V_{A}
$$


where $D_{A}=\left(\partial_{m}, D_{\alpha}, \bar{D}^{\alpha}\right)$ are super-covariant derivatives, see (A.3), and $V_{A}=$ $\left(V_{m}, V_{\alpha}, \bar{V}^{\alpha}\right)$ are gauge connections subject to the constraints

$$
\begin{aligned}
& \left\{\nabla_{\alpha}, \bar{\nabla}_{\beta}\right\}=-2 i\left(\gamma^{m}\right)_{\alpha \beta} \nabla_{m}+2 i \varepsilon_{\alpha \beta} G+2 \gamma_{\alpha \beta}^{3} H, \\
& {\left[\nabla_{\alpha}, \nabla_{m}\right]=-\left(\gamma_{m}\right)_{\alpha \beta} \bar{W}^{\beta}, \quad\left[\bar{\nabla}_{\alpha}, \nabla_{m}\right]=\left(\gamma_{m}\right)_{\alpha \beta} W^{\beta}} \\
& {\left[\nabla_{m}, \nabla_{n}\right]=i F_{m n} .}
\end{aligned}
$$

Here $G, H, W_{\alpha}$ and $F_{m n}$ are superfield strengths with the following conjugation properties

$$
G^{\dagger}=G, \quad H^{\dagger}=H, \quad\left(W_{\alpha}\right)^{\dagger}=\bar{W}_{\alpha}, \quad\left(F_{m n}\right)^{\dagger}=F_{m n} .
$$

In two dimensions, the antisymmetric tensor $F_{m n}$ has only one independent component, $F_{m n}=\varepsilon_{m n} f$, where for the antisymmetric $\varepsilon$-tensor $\varepsilon_{m n}=-\varepsilon_{n m}$ with vector indices we use the convention $\varepsilon_{01}=-\varepsilon^{01}=1$.

The algebra of covariant derivatives (2.2) implies a number of Bianchi identities. In particular, the scalar superfields $G$ and $H$ are covariantly linear,

$$
\nabla^{2} G=\nabla^{2} H=0, \quad \bar{\nabla}^{2} G=\bar{\nabla}^{2} H=0 .
$$

The spinor superfield strengths $W_{\alpha}$ and $\bar{W}_{\alpha}$ are expressed via the scalar ones,

$$
\bar{W}_{\alpha}=\nabla_{\alpha} G=-i\left(\gamma^{3}\right)_{\alpha}^{\beta} \nabla_{\beta} H, \quad W_{\alpha}=\bar{\nabla}_{\alpha} G=i\left(\gamma^{3}\right)_{\alpha}^{\beta} \bar{\nabla}_{\beta} H .
$$

As usual, they have the chirality properties

$$
\nabla_{\alpha} \bar{W}_{\beta}=0, \quad \bar{\nabla}_{\alpha} W_{\beta}=0
$$

and obey the 'standard' Bianchi identity

$$
\nabla^{\alpha} W_{\alpha}=\bar{\nabla}^{\alpha} \bar{W}_{\alpha}
$$

In its turn, the tensor field strength $F_{m n}$ is expressed via $W_{\alpha}$ and $\bar{W}_{\alpha}$,

$$
F_{m n} \equiv \varepsilon_{m n} f=-\frac{1}{4} \varepsilon_{m n}\left(\gamma^{3}\right)^{\alpha \beta}\left(\nabla_{\alpha} W_{\beta}-\bar{\nabla}_{\alpha} \bar{W}_{\beta}\right) .
$$

Another important relation appears by commuting (2.2a) with the superfield $G$ and applying properties (2.5)

$$
\nabla_{\alpha} W_{\beta}+\bar{\nabla}_{\alpha} \bar{W}_{\beta}=-2 i \gamma_{\alpha \beta}^{m} \nabla_{m} G+2 \gamma_{\alpha \beta}^{3}[H, G]+\varepsilon_{\alpha \beta} \nabla^{\gamma} W_{\gamma}
$$

The algebra of covariant derivatives (2.2) is invariant under the $\tau$-gauge transformations

$$
\nabla_{A} \rightarrow e^{i \tau(z)} \nabla_{A} e^{-i \tau(z)}
$$

with $\tau(z)$ being real gauge superfield parameter, $\tau^{\dagger}=\tau$.

The gauge connections $V_{A}$ may be expressed via a prepotential. In this paper we will use the real superfield prepotential $V$ which is introduced in such a way that the gauge-covariant spinor derivatives acquire the form (chiral representation)

$$
\nabla_{\alpha}=e^{-2 V} D_{\alpha} e^{2 V}, \quad \bar{\nabla}_{\alpha}=\bar{D}_{\alpha}
$$


In this case, as a consequence of (2.2a), the scalar superfield strengths are expressed via the prepotential as

$$
G=\frac{i}{4} \bar{D}^{\alpha}\left(e^{-2 V} D_{\alpha} e^{2 V}\right), \quad H=-\frac{1}{4}\left(\gamma^{3}\right)^{\alpha \beta} \bar{D}_{\beta}\left(e^{-2 V} D_{\alpha} e^{2 V}\right) .
$$

The expressions of the other superfield strengths in terms of $V$ can be obtained using (2.5) and (2.8). Note that all these superfield strengths transform covariantly under the $\Lambda$-gauge transformation of the prepotential

$$
e^{2 V} \rightarrow e^{i \bar{\Lambda}} e^{2 V} e^{-i \Lambda}
$$

with a chiral $\Lambda$.

The superfield strengths $G$ and $H$ can be considered as the real and imaginary parts of a complex superfield $\Sigma$ and its (Hermitian) conjugate $\bar{\Sigma}$

$$
\Sigma=G+i H, \quad \bar{\Sigma}=G-i H .
$$

From (2.5) it is easy to deduce twisted chirality properties of these superfields

$$
\nabla_{+} \Sigma=\bar{\nabla}_{-} \Sigma=0, \quad \bar{\nabla}_{+} \bar{\Sigma}=\nabla_{-} \bar{\Sigma}=0,
$$

where $\left(\nabla_{+}, \nabla_{-}\right) \equiv\left(\nabla_{1}, \nabla_{2}\right)$. The existence of such twisted chiral superfields is an important feature of two-dimensional gauge theory in superspace as compared with the higherdimensional cases. These superfield strengths play central role in superfield description of gauge theories in the $\mathcal{N}=(2,2)$ superspace.

\subsection{Parallel displacement propagator in $\mathcal{N}=(2,2)$ superspace}

In superspace, the parallel displacement propagator was introduced in the work [18] as a key ingredient which provides correct transformation properties of Green's functions and corresponding heat kernels under gauge transformations. This allowed the authors of [18] to develop a gauge-covariant procedure of perturbative computations of effective actions in supersymmetric gauge theories. In particular, this technique appeared very fruitful in the study of low-energy effective actions in different four-dimensional gauge theories in $\mathcal{N}=1$ and $\mathcal{N}=2$ superspaces [28-34]. For three-dimensional gauge theories this method was generalized in [23, 27]. This section is aimed at extending the basic concepts of the procedure of covariant perturbative computations to two-dimensional gauge theories in the $\mathcal{N}=(2,2)$ superspace.

Let us consider a superfield $\Phi$ in some representation $R$ of the gauge group, and its Hermitian conjugate $\bar{\Phi}$ transforming in the representation $\bar{R}$,

$$
\Phi(z) \rightarrow \Phi^{\prime}(z)=e^{i \tau(z)} \Phi(z), \quad \bar{\Phi}(z) \rightarrow \bar{\Phi}^{\prime}(z)=\bar{\Phi}(z) e^{-i \tau(z)},
$$

where $\tau=\tau^{\dagger}$ is Hermitian, but otherwise arbitrary gauge superfield parameter. Correspondingly, Green's function for these fields $G\left(z, z^{\prime}\right)=i\left\langle\Phi(z) \bar{\Phi}\left(z^{\prime}\right)\right\rangle$ has the transformation property

$$
G\left(z, z^{\prime}\right) \rightarrow e^{i \tau(z)} G\left(z, z^{\prime}\right) e^{-i \tau\left(z^{\prime}\right)}
$$


In a similar way, the parallel displacement propagator $I\left(z, z^{\prime}\right)$ is, by definition, a twopoint superspace function which transforms under the gauge group as

$$
I\left(z, z^{\prime}\right) \rightarrow e^{i \tau(z)} I\left(z, z^{\prime}\right) e^{-i \tau\left(z^{\prime}\right)} .
$$

Moreover, it is required to obey the differential equation

$$
\zeta^{A} \nabla_{A} I\left(z, z^{\prime}\right)=\zeta^{A}\left(D_{A}+V_{A}\right) I\left(z, z^{\prime}\right)=0
$$

and the boundary condition

$$
I(z, z)=\mathbf{1} .
$$

The latter means that at coincident superspace points $I\left(z, z^{\prime}\right)$ reduces to the identity operator in the gauge group. In eq. (2.19), $\zeta^{A} \equiv\left(\rho^{m}, \zeta^{\alpha}, \bar{\zeta}_{\alpha}\right)$ is the $\mathcal{N}=(2,2)$ supersymmetric interval with the components

$$
\rho^{m}=\left(x-x^{\prime}\right)^{m}-i\left(\theta-\theta^{\prime}\right)^{\alpha} \gamma_{\alpha \beta}^{m} \bar{\theta}^{\prime \beta}+i \theta^{\prime \alpha} \gamma_{\alpha \beta}^{m}\left(\bar{\theta}-\bar{\theta}^{\prime}\right)^{\beta}, \quad \zeta^{\alpha}=\left(\theta-\theta^{\prime}\right)^{\alpha}, \quad \bar{\zeta}_{\alpha}=\left(\bar{\theta}-\bar{\theta}^{\prime}\right)_{\alpha} .
$$

It is possible to show that the properties (2.18) and (2.20) imply the important relation

$$
I\left(z, z^{\prime}\right) I\left(z^{\prime}, z\right)=\mathbf{1} .
$$

Note also that the rule of Hermitian conjugation for $I\left(z, z^{\prime}\right)$ looks like

$$
\left(I\left(z, z^{\prime}\right)\right)^{\dagger}=I\left(z^{\prime}, z\right) .
$$

The algebra of covariant derivatives (2.2) can be represented in the condensed form

$$
\left[\nabla_{A}, \nabla_{B}\right\}=\mathbf{T}_{A B}{ }^{C} \nabla_{C}+i \mathbf{F}_{A B}
$$

where $\mathbf{T}_{A B}{ }^{C}$ is the supertorsion and $\mathbf{F}_{A B}$ is the field strength for gauge superfield connection (2.1). The non-vanishing components of these tensors can be read off from (2.2). They appear in the following important relation for the derivative of the parallel displacement propagator [18]

$$
\begin{gathered}
\nabla_{B} I\left(z, z^{\prime}\right)=i \sum_{n=1}^{\infty} \frac{(-1)^{n}}{(n+1) !}\left[-\zeta^{A_{n}} \ldots \zeta^{A_{1}} \nabla_{A_{1}} \ldots \nabla_{A_{n-1}} \mathbf{F}_{A_{n} B}(z)\right. \\
\left.\quad+\frac{(n-1)}{2} \zeta^{A_{n}} \mathbf{T}_{A_{n} B}{ }^{C} \zeta^{A_{n-1}} \ldots \zeta^{A_{1}} \nabla_{A_{1}} \ldots \nabla_{A_{n-2}} \mathbf{F}_{A_{n-1} C}(z)\right] I\left(z, z^{\prime}\right) .
\end{gathered}
$$

This identity shows that any covariant derivative of the parallel displacement propagator may be expressed in terms of the parallel displacement propagator itself and covariant derivatives of the superfield strength together with the torsion tensor. This identity appears crucial in perturbative computations of low-energy effective action which is a functional of these tensors.

In general, (2.25) is an infinite series over covariant derivatives of the field strength $\mathbf{F}_{A B}$. It is natural to expect that for certain field configurations this series terminates. 
In particular, it is possible to show that for the covariantly constant vector multiplet background

$$
\nabla_{m} \Sigma=\nabla_{m} \bar{\Sigma}=0, \quad \nabla_{m} W_{\alpha}=\nabla_{m} \bar{W}_{\alpha}=0
$$

the identity (2.25) reduces to

$$
\begin{aligned}
& \nabla_{\beta} I\left(z, z^{\prime}\right)=\left[-i \bar{\zeta}_{\beta} G+\left(\gamma^{3}\right)_{\beta}^{\alpha} \bar{\zeta}_{\alpha} H+\frac{1}{6}\left(\gamma^{m}\right)_{\beta \alpha}\left(3 \rho_{m} \bar{W}^{\alpha}-\rho_{m} \bar{\zeta}_{\gamma} \bar{\nabla}^{\gamma} \bar{W}^{\alpha}-\bar{\zeta}^{\alpha} \rho^{n} F_{n m}\right)\right. \\
& +\frac{i}{6} \bar{\zeta}^{2} W_{\beta}+\frac{i}{6} \bar{\zeta}_{\beta} \zeta^{\alpha} \bar{W}_{\alpha}-\frac{i}{3} \bar{\zeta}^{\alpha} \zeta_{\alpha} \bar{W}_{\beta}-\frac{i}{2} \bar{\zeta}_{\alpha} \zeta^{\gamma}\left(\gamma^{3}\right)_{\beta}^{\alpha}\left(\gamma^{3}\right)_{\gamma}^{\delta} \bar{W}_{\delta} \\
& \left.+\frac{i}{6} \bar{\zeta}^{2} \zeta^{\alpha} \bar{\nabla}_{(\alpha} \bar{W}_{\beta)}\right] I\left(z, z^{\prime}\right) \\
& \bar{\nabla}^{\beta} I\left(z, z^{\prime}\right)=\left[-i \zeta^{\beta} G+\left(\gamma^{3}\right)_{\alpha}^{\beta} \zeta^{\alpha} H+\frac{1}{6}\left(\gamma^{m}\right)^{\beta \alpha}\left(3 \rho_{m} W_{\alpha}-\rho_{m} \zeta^{\gamma} \nabla_{\gamma} W_{\alpha}-\zeta_{\alpha} \rho^{n} F_{n m}\right)\right. \\
& -\frac{i}{6} \zeta^{2} \bar{W}^{\beta}-\frac{i}{6} \zeta^{\beta} \bar{\zeta}^{\alpha} W_{\alpha}+\frac{i}{3} \zeta^{\alpha} \bar{\zeta}_{\alpha} W^{\beta}-\frac{i}{2} \zeta^{\alpha} \bar{\zeta}_{\gamma}\left(\gamma^{3}\right)_{\alpha}^{\beta}\left(\gamma^{3}\right)_{\delta}^{\gamma} W^{\delta} \\
& \left.-\frac{i}{6} \zeta^{2} \bar{\zeta}_{\alpha} \nabla^{(\alpha} W^{\beta)}\right] I\left(z, z^{\prime}\right) \\
& \nabla_{m} I\left(z, z^{\prime}\right)=\left[\frac{i}{2} \rho^{n} F_{n m}-\frac{1}{2}\left(\gamma_{m}\right)_{\alpha \beta}\left(\zeta^{\alpha} \bar{W}^{\beta}+\bar{\zeta}^{\alpha} W^{\beta}-\frac{1}{3} \zeta^{\alpha} \bar{\zeta}_{\gamma} \bar{\nabla}^{\gamma} \bar{W}^{\beta}\right.\right. \\
& \left.\left.+\frac{1}{3} \bar{\zeta}^{\alpha} \zeta_{\gamma} \nabla^{\gamma} W^{\beta}\right)\right] I\left(z, z^{\prime}\right)
\end{aligned}
$$

As we will show in the following subsections, these identities appear very useful in computing heat kernels of Green's functions of various operators in the $\mathcal{N}=(2,2)$ superspace.

\subsection{Real superfield Green's function and its heat kernel}

The real superfield d'Alembertian is defined by either expression

$$
\begin{aligned}
\square_{\mathrm{v}} & =\frac{1}{16}\left\{\nabla^{2}, \bar{\nabla}^{2}\right\}-\frac{1}{8} \nabla^{\alpha} \bar{\nabla}^{2} \nabla_{\alpha}+\frac{i}{2}\left(\nabla^{\alpha} W_{\alpha}\right)+i W^{\alpha} \nabla_{\alpha} \\
& =\frac{1}{16}\left\{\nabla^{2}, \bar{\nabla}^{2}\right\}-\frac{1}{8} \bar{\nabla}^{\alpha} \nabla^{2} \bar{\nabla}_{\alpha}-\frac{i}{2}\left(\bar{\nabla}^{\alpha} \bar{W}_{\alpha}\right)-i \bar{W}^{\alpha} \bar{\nabla}_{\alpha}
\end{aligned}
$$

By making use of the algebra (2.2), this operator may be brought to the form

$$
\square_{\mathrm{v}}=\nabla^{m} \nabla_{m}+\frac{1}{2}\{\Sigma, \bar{\Sigma}\}+i W^{\alpha} \nabla_{\alpha}-i \bar{W}^{\alpha} \bar{\nabla}_{\alpha} .
$$

Green's function $G_{\mathrm{V}}\left(z, z^{\prime}\right)$ of this operator is defined as a solution of the equation

$$
\left(\square_{\mathrm{v}}+m^{2}\right) G_{\mathrm{v}}\left(z, z^{\prime}\right)=-\delta^{2 \mid 4}\left(z-z^{\prime}\right),
$$

where $m$ is a mass parameter and $\delta^{2 \mid 4}\left(z-z^{\prime}\right)$ is the full superspace delta function,

$$
\delta^{2 \mid 4}\left(z-z^{\prime}\right)=\delta^{2}\left(x-x^{\prime}\right) \delta^{4}\left(\theta-\theta^{\prime}\right) .
$$

Green's function can be represented as a proper-time integral of the corresponding heat kernel $K_{\mathrm{v}}\left(z, z^{\prime} \mid s\right)$

$$
G_{\mathrm{v}}\left(z, z^{\prime}\right)=-i \int_{0}^{\infty} d s K_{\mathrm{v}}\left(z, z^{\prime} \mid s\right) e^{-s\left(\epsilon+i m^{2}\right)}
$$


where $\epsilon \rightarrow+0$ implements standard boundary condition for the propagator. The equation for the propagator (2.30) is satisfied when the heat kernel obeys the conditions

$$
\left(i \frac{d}{d s}-\square_{\mathrm{v}}\right) K_{\mathrm{v}}\left(z, z^{\prime} \mid s\right)=0, \quad \lim _{s \rightarrow 0} K_{\mathrm{v}}\left(z, z^{\prime} \mid s\right)=\delta^{2 \mid 4}\left(z-z^{\prime}\right) .
$$

In general, it is very hard to solve these equations explicitly. Nevertheless, it is possible to find the exact solution for the heat kernel when the background gauge superfield obeys the following two constraints:

i) Gauge multiplet obeys super Yang-Mills equations of motion (on-shell background)

$$
\nabla^{\alpha} W_{\alpha}=0, \quad \bar{\nabla}^{\alpha} \bar{W}_{\alpha}=0 ;
$$

ii) Field strengths are covariantly constant

$$
\nabla_{m} \Sigma=\nabla_{m} \bar{\Sigma}=0, \quad \nabla_{m} W_{\alpha}=\nabla_{m} \bar{W}_{\alpha}=0 .
$$

It is important to note that the compatibility condition for the constraint (2.35) requires that the background gauge superfield belongs to the Cartan subalgebra of the Lie algebra of the gauge group. This means that the background gauge superfields are (anti)commuting.

The procedure of solving the heat kernel equation for the covariantly constant vector multiplet background was developed in the four-dimensional case in [17-20] and successfully applied for three-dimensional gauge theories in [23-25]. In the two-dimensional case the same procedure yields

$$
K_{\mathrm{v}}\left(z, z^{\prime} \mid s\right)=\frac{1}{4 \pi s} \frac{s f}{\sinh (s f)} e^{-i s \Sigma \bar{\Sigma}} \mathcal{O}(s) e^{-\frac{i}{4}(f \operatorname{coth} s f) \rho^{m} \rho_{m}} \zeta^{2} \bar{\zeta}^{2} I\left(z, z^{\prime}\right),
$$

where $\zeta^{2}=\zeta^{\alpha} \zeta_{\alpha}, \bar{\zeta}^{2}=\bar{\zeta}^{\alpha} \bar{\zeta}_{\alpha}$ and $\mathcal{O}(s)$ is the 'shift' operator

$$
\mathcal{O}(s)=e^{s\left(W^{\alpha} \nabla_{\alpha}-\bar{W}^{\alpha} \bar{\nabla}_{\alpha}\right)} .
$$

Within quantum loop computations, it is often necessary to know the value of the heat kernel at coincident superspace points. For this aim, it is useful to have such a representation for the heat kernel (2.36) where the operator $\mathcal{O}(s)$ appears on the right and hits the parallel displacement propagator,

$$
K_{\mathrm{v}}\left(z, z^{\prime} \mid s\right)=\frac{1}{4 \pi s} \frac{s f}{\sinh (s f)} e^{-i s \Sigma \bar{\Sigma}} e^{-\frac{i}{4}(f \operatorname{coth} s f) \zeta^{m}(s) \zeta_{m}(s)} \zeta^{2}(s) \bar{\zeta}^{2}(s) I\left(z, z^{\prime} \mid s\right) .
$$

Here the operator $\mathcal{O}(s)$ is used to define the $s$-dependent superfield strengths and components of the superspace interval

$$
\begin{aligned}
W^{\alpha}(s) & \equiv \mathcal{O}(s) W^{\alpha} \mathcal{O}(-s)=W^{\beta}\left(e^{s N}\right)_{\beta}{ }^{\alpha} \\
\zeta^{\alpha}(s) & \equiv \mathcal{O}(s) \zeta^{\alpha} \mathcal{O}(-s)=\zeta^{\alpha}+W^{\beta}\left(\left(e^{s N}-1\right) N^{-1}\right)_{\beta}{ }^{\alpha} \\
\bar{\zeta}^{\alpha}(s) & \equiv \mathcal{O}(s) \bar{\zeta}^{\alpha} \mathcal{O}(-s)=\bar{\zeta}^{\alpha}-\bar{W}^{\beta}\left(\left(e^{-s \bar{N}}-1\right) \bar{N}^{-1}\right)_{\beta}{ }^{\alpha} \\
\rho^{m}(s) & \equiv \mathcal{O}(s) \rho^{m} \mathcal{O}(-s)=\rho^{m}+i\left(\gamma^{m}\right)^{\alpha \beta} \int_{0}^{s} d t\left(W_{\alpha}(t) \bar{\zeta}_{\beta}(t)+\bar{W}_{\alpha}(t) \zeta_{\beta}(t)\right)
\end{aligned}
$$


and

$$
I\left(z, z^{\prime} \mid s\right) \equiv \mathcal{O}(s) I\left(z, z^{\prime}\right) .
$$

In formulas (2.39) we have introduced the notation

$$
N_{\alpha \beta}=\nabla_{(\alpha} W_{\beta)}, \quad \bar{N}_{\alpha \beta}=\bar{\nabla}_{(\alpha} \bar{W}_{\beta)} .
$$

The $s$-dependent parallel displacement propagator (2.40) can be represented in the following form [18]

$$
I\left(z, z^{\prime} \mid s\right)=\exp \left[\int_{0}^{s} d t \Xi\left(z, z^{\prime} \mid t\right)\right] I\left(z, z^{\prime}\right)
$$

where

$$
\Xi\left(z, z^{\prime} \mid t\right)=\mathcal{O}(t) \Xi\left(z, z^{\prime}\right) \mathcal{O}(-t)
$$

and $\Xi\left(z, z^{\prime}\right)$ solves for

$$
\left(W^{\alpha} \nabla_{\alpha}-\bar{W}^{\alpha} \bar{\nabla}_{\alpha}\right) I\left(z, z^{\prime}\right)=\Xi\left(z, z^{\prime}\right) I\left(z, z^{\prime}\right) .
$$

Making use of (2.27a) and (2.27b) we find

$$
\begin{aligned}
\Xi\left(z, z^{\prime}\right)= & i\left(\zeta^{\alpha} \bar{W}_{\beta}+\bar{\zeta}_{\beta} W^{\alpha}\right)\left(\delta_{\alpha}^{\beta} G+i\left(\gamma^{3}\right)_{\alpha}^{\beta} H\right) \\
& +\frac{i}{6} \bar{\zeta}^{2}\left(W^{2}+\zeta^{\alpha} W^{\beta} \nabla_{\alpha} W_{\beta}\right)+\frac{i}{6} \zeta^{2}\left(\bar{W}^{2}+\bar{\zeta}^{\alpha} \bar{W}^{\beta} \nabla_{\alpha} W_{\beta}\right) \\
& +\frac{4 i}{3} \zeta^{\alpha} \bar{\zeta}^{\beta} W_{\alpha} \bar{W}_{\beta}-i \zeta^{\alpha} \bar{\zeta}_{\alpha} W^{\beta} \bar{W}_{\beta}+i \zeta^{\gamma} \bar{\zeta}_{\delta}\left(\gamma^{3}\right)_{\beta}^{\alpha}\left(\gamma^{3}\right)_{\gamma}^{\delta} W^{\beta} \bar{W}_{\alpha}
\end{aligned}
$$

The expression for $\Xi\left(z, z^{\prime} \mid s\right)$ can be found from the above formula just by replacing all superfield strengths and components of the superspace interval by the corresponding $s$ dependent quantities from (2.39).

\subsection{Heat kernel for chiral superfield Green's function}

Consider gauge-covariant chiral superfield $\Phi, \bar{\nabla}_{\alpha} \Phi=0$, and its Hermitian conjugate $\bar{\Phi}$. The d'Alembertian operators acting in the space of such fields are defined in the standard way

$$
\square_{+} \Phi=\frac{1}{16} \bar{\nabla}^{2} \nabla^{2} \Phi, \quad \square_{-} \bar{\Phi}=\frac{1}{16} \nabla^{2} \bar{\nabla}^{2} \bar{\Phi} .
$$

Making use of the algebra of covariant derivatives (2.2) one uncovers the following representations for these operators

$$
\begin{aligned}
& \square_{+}=\frac{1}{16} \bar{\nabla}^{2} \nabla^{2}=\nabla^{m} \nabla_{m}+\frac{1}{2}\{\Sigma, \bar{\Sigma}\}+\frac{i}{2}\left(\nabla^{\alpha} W_{\alpha}\right)+i W^{\alpha} \nabla_{\alpha}, \\
& \square_{-}=\frac{1}{16} \nabla^{2} \bar{\nabla}^{2}=\nabla^{m} \nabla_{m}+\frac{1}{2}\{\Sigma, \bar{\Sigma}\}-\frac{i}{2}\left(\bar{\nabla}^{\alpha} \bar{W}_{\alpha}\right)-i \bar{W}^{\alpha} \bar{\nabla}_{\alpha} .
\end{aligned}
$$

By definition, Green's functions for these operators and the corresponding propagators obey

$$
\begin{array}{rlrl}
i\left\langle\Phi(z) \Phi^{\mathrm{T}}\left(z^{\prime}\right)\right\rangle & =-m G_{+}\left(z, z^{\prime}\right), & & \left(\square_{+}+m^{2}\right) G_{+}\left(z, z^{\prime}\right)=-\delta_{+}^{2 \mid 2}\left(z, z^{\prime}\right), \\
i\left\langle\bar{\Phi}^{\mathrm{T}}(z) \bar{\Phi}\left(z^{\prime}\right)\right\rangle=m G_{-}\left(z, z^{\prime}\right), & & \left(\square_{-}+m^{2}\right) G_{-}\left(z, z^{\prime}\right)=-\delta_{-}^{2 \mid 2}\left(z, z^{\prime}\right),
\end{array}
$$


where $\delta_{ \pm}^{2 \mid 2}\left(z, z^{\prime}\right)$ are (anti)chiral delta-functions which are related to the full superspace delta-function (2.31) as

$$
\delta_{+}^{2 \mid 2}\left(z, z^{\prime}\right)=-\frac{1}{4} \bar{\nabla}^{2} \delta^{2 \mid 4}\left(z-z^{\prime}\right), \quad \delta_{-}^{2 \mid 2}\left(z, z^{\prime}\right)=-\frac{1}{4} \nabla^{2} \delta^{2 \mid 4}\left(z-z^{\prime}\right) .
$$

For Green's functions (2.48) there are the associated heat kernels

$$
G_{ \pm}\left(z, z^{\prime}\right)=-i \int_{0}^{\infty} d s K_{ \pm}\left(z, z^{\prime} \mid s\right) e^{-s\left(\epsilon+i m^{2}\right)}, \quad \epsilon \rightarrow+0 .
$$

It is known $[18,28]$ that for the on-shell vector multiplet background (2.34) the chiral Green functions $G_{ \pm}$are related to $G_{\mathrm{v}}$ as

$$
G_{+}\left(z, z^{\prime}\right)=-\frac{1}{4} \bar{\nabla}^{2} G_{\mathrm{v}}\left(z, z^{\prime}\right), \quad G_{-}\left(z, z^{\prime}\right)=-\frac{1}{4} \nabla^{2} G_{\mathrm{v}}\left(z, z^{\prime}\right) .
$$

It is easy to check these relations using the identities

$$
\begin{array}{rlrl}
\nabla^{2} \square_{+} & =\square_{-} \nabla^{2}, & & \bar{\nabla}^{2} \square_{-}=\square_{+} \bar{\nabla}^{2}, \\
\nabla^{2} \square_{+}=\nabla^{2} \square_{\mathrm{v}}=\square_{\mathrm{v}} \nabla^{2}, & & \bar{\nabla}^{2} \square_{-}=\bar{\nabla}^{2} \square_{\mathrm{v}}=\square_{\mathrm{v}} \bar{\nabla}^{2} .
\end{array}
$$

It should be noted that the identities (2.53) hold only for the on-shell vector multiplet background (2.34). The equations (2.51) imply similar relations for the corresponding heat kernels

$$
K_{+}\left(z, z^{\prime} \mid s\right)=-\frac{1}{4} \bar{\nabla}^{2} K_{\mathrm{v}}\left(z, z^{\prime} \mid s\right), \quad K_{-}\left(z, z^{\prime} \mid s\right)=-\frac{1}{4} \nabla^{2} K_{\mathrm{v}}\left(z, z^{\prime} \mid s\right) .
$$

Thus, the computation of the heat kernels $K_{ \pm}$is reduced to finding the result of the action of the operators $\nabla^{2}$ and $\bar{\nabla}^{2}$ on the heat kernel (2.36).

It is possible to show that upon acting by $\bar{\nabla}^{2}$ on (2.36), this operator hits only $\zeta^{2} \bar{\zeta}^{2} I\left(z, z^{\prime}\right)$ since the factor in front of this function originates from $e^{-i s \square_{\mathrm{v}}}$. The latter operator commutes with $\bar{\nabla}^{2}$ owing to the identities (2.53). Thus, for $K_{+}$we have

$$
K_{+}\left(z, z^{\prime} \mid s\right)=\frac{1}{4 \pi s} \frac{s f}{\sinh (s f)} e^{-i s \Sigma \bar{\Sigma}} \mathcal{O}(s) e^{-\frac{i}{4}(f \operatorname{coth} s f) \rho^{m} \rho_{m}} \zeta^{2}\left(-\frac{1}{4} \bar{\nabla}^{2}\right) \bar{\zeta}^{2} I\left(z, z^{\prime}\right) .
$$

Applying (2.27b) we compute the action of the operator $\bar{\nabla}^{2}$ on the parallel displacement propagator

$$
-\frac{1}{4} \zeta^{2} \bar{\nabla}^{2}\left(\bar{\zeta}^{2} I\left(z, z^{\prime}\right)\right)=\zeta^{2} e^{-\frac{1}{2}\left(\gamma^{m}\right)_{\alpha \beta} \rho_{m} \bar{\zeta}^{\alpha} W^{\beta}} I\left(z, z^{\prime}\right) .
$$

Substituting this identity into (2.55) we find

$$
\begin{aligned}
K_{+}\left(z, z^{\prime} \mid s\right)= & \frac{1}{4 \pi s} \frac{s f}{\sinh (s f)} e^{-i s \Sigma \bar{\Sigma}} e^{-\frac{i}{4}(f \operatorname{coth} s f) \rho^{m}(s) \rho_{m}(s)-\frac{1}{2}\left(\gamma^{m}\right)_{\alpha \beta} \rho_{m}(s) \bar{\zeta}^{\alpha}(s) W^{\beta}(s)} \\
& \times \zeta^{2}(s) I\left(z, z^{\prime} \mid s\right)
\end{aligned}
$$

Here we pushed the operator $\mathcal{O}(s)$ through on the right that resulted in making all objects $s$-dependent according to (2.39) and (2.40). In a similar way we find the antichiral heat kernel

$$
\begin{aligned}
K_{-}\left(z, z^{\prime} \mid s\right)= & \frac{1}{4 \pi s} \frac{s f}{\sinh (s f)} e^{-i s \Sigma \bar{\Sigma}} e^{-\frac{i}{4}(f \operatorname{coth} s f) \rho^{m}(s) \rho_{m}(s)-\frac{1}{2}\left(\gamma^{m}\right){ }_{\alpha \beta} \rho_{m}(s) \zeta^{\alpha}(s) \bar{W}^{\beta}(s)} \\
& \times \bar{\zeta}^{2}(s) I\left(z, z^{\prime} \mid s\right)
\end{aligned}
$$


We point out that the expressions for the (anti)chiral heat kernels are very similar to the ones in the four-dimensional supersymmetric gauge theory [28].

\subsection{Heat kernel for Green's function $G_{+-}$}

Finally, we consider the propagators among chiral and antichiral superfields

$$
i\left\langle\Phi(z) \bar{\Phi}\left(z^{\prime}\right)\right\rangle=G_{+-}\left(z, z^{\prime}\right), \quad i\left\langle\bar{\Phi}(z) \Phi\left(z^{\prime}\right)\right\rangle=G_{-+}\left(z, z^{\prime}\right) .
$$

By definition, these Green's functions obey

$$
\begin{aligned}
& \frac{1}{4} \nabla^{2} G_{+-}\left(z, z^{\prime}\right)+m^{2} G_{-}\left(z, z^{\prime}\right)=-\delta_{-}^{2 \mid 2}\left(z, z^{\prime}\right), \\
& \frac{1}{4} \bar{\nabla}^{2} G_{-+}\left(z, z^{\prime}\right)+m^{2} G_{+}\left(z, z^{\prime}\right)=-\delta_{+}^{2 \mid 2}\left(z, z^{\prime}\right) .
\end{aligned}
$$

With Green's functions (2.59) are associated the corresponding heat kernels

$$
\begin{aligned}
& G_{+-}\left(z, z^{\prime}\right)=-i \int_{0}^{\infty} d s K_{+-}\left(z, z^{\prime} \mid s\right) e^{-s\left(\epsilon+i m^{2}\right)}, \\
& G_{-+}\left(z, z^{\prime}\right)=-i \int_{0}^{\infty} d s K_{-+}\left(z, z^{\prime} \mid s\right) e^{-s\left(\epsilon+i m^{2}\right)} .
\end{aligned}
$$

This subsection aims to find explicit solutions for these heat kernels on the covariantly constant vector multiplet background.

First of all, we point out that, as a consequence of the definitions of covariantly (anti)chiral d'Alembertian operators (2.47), Green's functions (2.59) are related to the (anti)chiral ones (2.48) as

$$
G_{+-}\left(z, z^{\prime}\right)=\frac{1}{4} \bar{\nabla}^{2} G_{-}\left(z, z^{\prime}\right), \quad G_{-+}\left(z, z^{\prime}\right)=\frac{1}{4} \nabla^{2} G_{+}\left(z, z^{\prime}\right) .
$$

Analogous relations hold for the corresponding heat kernels

$$
K_{+-}\left(z, z^{\prime} \mid s\right)=\frac{1}{4} \bar{\nabla}^{2} K_{-}\left(z, z^{\prime} \mid s\right), \quad K_{-+}\left(z, z^{\prime} \mid s\right)=\frac{1}{4} \nabla^{2} K_{+}\left(z, z^{\prime} \mid s\right) .
$$

Thus, the problem is reduced to finding the action of the operators $\nabla^{2}$ and $\bar{\nabla}^{2}$ on the heat kernels (2.57) and (2.58).

Let us consider the derivation of the heat kernel $K_{+-}$in some details. It appears upon acting by the operator $\bar{\nabla}^{2}$ on the heat kernel (2.58). Note that, owing to (2.53), this operator commutes with the expression $e^{-i s \Sigma \Sigma \bar{\Sigma}} e^{-\frac{i}{4}(f \operatorname{coth} s f) \rho^{m} \rho_{m}}$ since the latter originates from $e^{-i s \square_{\mathrm{v}}}$. Thus, we need to find the action of this operator on the rest using the properties of the parallel displacement operator $(2.27 \mathrm{~b})$

$$
-\frac{1}{4} \bar{\nabla}^{2}\left(e^{-\frac{1}{2}\left(\gamma^{m}\right)_{\alpha \beta} \rho_{m} \zeta^{\alpha} \bar{W}^{\beta}} \bar{\zeta}^{2} I\left(z, z^{\prime}\right)\right)=e^{R\left(z, z^{\prime}\right)} I\left(z, z^{\prime}\right),
$$

where

$$
\begin{aligned}
R\left(z, z^{\prime}\right)= & -i \zeta^{\alpha} \bar{\zeta}_{\alpha} G+\zeta^{\alpha} \bar{\zeta}_{\beta}\left(\gamma^{3}\right)_{\alpha}^{\beta} H-\frac{1}{2}\left(\gamma_{m}\right)_{\alpha \beta} \tilde{\rho}^{m}\left(\zeta^{\alpha} \bar{W}^{\beta}+\bar{\zeta}^{\alpha} W^{\beta}\right)+\frac{2 i}{3} \bar{\zeta}^{2} \zeta^{\alpha} W_{\alpha} \\
& -\frac{i}{6} \zeta^{2} \bar{\zeta}^{\alpha} \bar{W}_{\alpha}+\frac{1}{2}\left(\gamma_{m}\right)^{\alpha \beta} \tilde{\rho}^{m} \zeta_{\beta} \bar{\zeta}^{\gamma} \nabla_{\alpha} W_{\gamma}
\end{aligned}
$$


Here

$$
\tilde{\rho}^{m}=\rho^{m}+i \zeta^{\alpha} \gamma_{\alpha \beta}^{m} \bar{\zeta}^{\beta}
$$

is a modification of the supersymmetric interval which is chiral with respect to the first argument and antichiral with respect to the other

$$
D_{\alpha}^{\prime} \tilde{\rho}^{m}=\bar{D}_{\alpha} \tilde{\rho}^{m}=0
$$

Given the function $R\left(z, z^{\prime}\right)$ in the form (2.65), we have the following representation for the heat kernel $K_{+-}$

$$
K_{+-}\left(z, z^{\prime} \mid s\right)=-\frac{1}{4 \pi s} \frac{s f}{\sinh (s f)} e^{-i s \Sigma \bar{\Sigma}} \mathcal{O}(s) e^{-\frac{i}{4}(f \operatorname{coth} s f) \tilde{\rho}^{m} \tilde{\rho}_{m}+R\left(z, z^{\prime}\right)} I\left(z, z^{\prime}\right) .
$$

As the final step, in (2.68) we have to push the operator $\mathcal{O}(s)$ through on the right and hit the parallel displacement propagator according to eq. (2.40). This procedure effectively makes the superfield strengths and components of supersymmetric interval $s$-dependent according to (2.39)

$$
K_{+-}\left(z, z^{\prime} \mid s\right)=-\frac{1}{4 \pi s} \frac{s f}{\sinh (s f)} e^{-i s \Sigma \bar{\Sigma}} e^{-\frac{i}{4}(f \operatorname{coth} s f) \tilde{\rho}^{m}(s) \tilde{\rho}_{m}(s)+R\left(z, z^{\prime} \mid s\right)+\int_{0}^{s} d t \Xi(t)} I\left(z, z^{\prime}\right),
$$

where $R\left(z, z^{\prime} \mid s\right)=\mathcal{O}(s) R\left(z, z^{\prime}\right) \mathcal{O}(-s)$, and $\Xi(s)$ is given by (2.45). For practical computations, it is useful to represent the heat kernel (2.69) in the equivalent form

$$
K_{+-}\left(z, z^{\prime} \mid s\right)=-\frac{1}{4 \pi s} \frac{s f}{\sinh (s f)} e^{-i s \Sigma \bar{\Sigma}} e^{-\frac{i}{4}(f \operatorname{coth} s f) \tilde{\rho}^{m}(s) \tilde{\rho}_{m}(s)+R\left(z, z^{\prime}\right)+\int_{0}^{s} d t\left(R^{\prime}(t)+\Xi(t)\right)} I\left(z, z^{\prime}\right) .
$$

Here, the function $R^{\prime}(t)$ can be found explicitly from (2.65) using $R^{\prime}(t)=\mathcal{O}(t)\left[W^{\alpha} \nabla_{\alpha}-\right.$ $\left.\bar{W}^{\alpha} \bar{\nabla}_{\alpha}, R\right] \mathcal{O}(-t)$ and combined with (2.45):

$$
\begin{aligned}
R^{\prime}(t)+\Xi(t)= & \mathcal{O}(t)\left[2 i \bar{\zeta}_{\alpha} W^{\alpha} G-2\left(\gamma^{3}\right)_{\alpha}^{\beta} \bar{\zeta}_{\beta} W^{\alpha} H+\frac{5 i}{3} \zeta^{\alpha} W_{\alpha} \bar{\zeta}^{\beta} \bar{W}_{\beta}\right. \\
& +2 i \zeta^{\alpha} \bar{W}_{\alpha} \bar{\zeta}^{\beta} W_{\beta}-\frac{i}{6} \bar{\zeta}^{2} W^{2}+2 i\left(\gamma^{3}\right)_{\alpha}^{\beta}\left(\gamma^{3}\right)_{\gamma}^{\delta} \zeta^{\gamma} \bar{\zeta}_{\delta} W^{\alpha} \bar{W}_{\beta} \\
& \left.-\frac{11 i}{12} \bar{\zeta}^{2} \zeta^{\beta} \nabla_{\beta} W^{2}+\frac{1}{2}\left(\gamma_{m}\right)^{\alpha \beta} \tilde{\rho}^{m} \bar{\zeta}_{\alpha} \nabla_{\beta} W^{2}\right] \mathcal{O}(-t) .
\end{aligned}
$$

We point out that, as follows from (2.65), the function $R\left(z, z^{\prime}\right)$ vanishes at coincident Grassmann coordinates, $\left.R\left(z, z^{\prime}\right)\right|_{\zeta \rightarrow 0} \rightarrow 0$. However, the contribution from (2.71) is nontrivial at coincident points.

\section{Low-energy effective action in $\mathcal{N}=(2,2)$ SQED}

\subsection{General remarks}

In general, Abelian gauge theories in $\mathcal{N}=(2,2)$ superspace may include the following terms in the classical action: 
- The kinetic term for the vector multiplet $V$

$$
S_{V}=\frac{1}{2 e^{2}} \int d^{2 \mid 4} z \bar{\Sigma} \Sigma .
$$

Here $e$ is the dimensional gauge coupling, $[e]=1$, and $d^{2 \mid 4} z$ is the measure in the full $\mathcal{N}=(2,2)$ superspace (see appendix for our superspace conventions).

- The mass term for the vector multiplet

$$
S_{\mathfrak{m}}=-\frac{i}{4} \frac{\mathfrak{m}}{e^{2}} \int d^{2 \mid 2} \tilde{z} \Sigma^{2}+\text { c.c. },
$$

where the integration goes over the twisted chiral subspace and $\mathfrak{m}$ is, in general, complex mass parameter. Without loss of generality, we can set it to be real, $\overline{\mathfrak{m}}=\mathfrak{m}$, just to simplify some formulas below. Note that the sum of actions (3.1) and (3.2) amounts to the massive Wess-Zumino model for the twisted chiral multiplet $\Sigma$. It should be noted that the mass term (3.2) may be obtained by the dimensional reduction from the $3 \mathrm{~d} \mathcal{N}=2$ Chern-Simons action which plays role of the topological mass term in three-dimensional electrodynamics.

- Fayet-Iliopoulos (FI) term

$$
S_{\mathrm{FI}}=-\frac{i t}{2} \int d^{2 \mid 2} \tilde{z} \Sigma+\text { c.c. }
$$

where

$$
t=r+i \theta .
$$

In (3.3), the real part of the FI parameter $r$ couples with the auxiliary field $D$ of the vector multiplet while the imaginary part $\theta$ corresponds to the topological $f$-term and quantizes $[3,35]$.

- $N$ charged chiral multiplets $Q_{i}$ with charges $q_{i}$ and mass matrix $m_{i j}$

$$
S_{Q}=-\sum_{i=1}^{N} \int d^{2 \mid 4} z \bar{Q}_{i} e^{2 q_{i} V} Q_{i}-\sum_{i, j=1}^{N}\left(\int d^{2 \mid 2} z m_{i j} Q_{i} Q_{j}+\text { c.c. }\right) .
$$

Needless to say that the mass matrix $m_{i j}$ should be such that the gauge invariance is preserved. Chiral multiplets may also have real mass which originates from vevs of scalars in the vector multiplet $V$.

More generally, it is also possible to study quantum dynamics of twisted chiral multiplets as well as semi-chiral ones $[4,8,36,37]$, but such models are beyond the scope of this paper.

Depending on the number of chiral multiplets and on the values of all mentioned above parameters, Abelian gauge theories in the $\mathcal{N}=(2,2)$ superspace exhibit different phases which are thoroughly investigated in [3]. In this paper, we are interested in the effective action on the Coulomb branch. It is known that the necessary condition for existence of 
the Coulomb branch at the quantum level is that the charges of all chiral multiplets should sum to zero

$$
\sum_{i=1}^{N} q_{i}=0 .
$$

Indeed, when this condition is not satisfied, the following two effects occur: i) There are UV-divergent tadpole Feynman graphs which result in the renormalization of the FI parameter. These quantum corrections lift the Coulomb branch. ii) The effective twisted superpotential for the superfield strength $\Sigma$ is generated at one loop [2]. This effective superpotential may also be interpreted as a functional reproducing correct transformation properties of the effective action under anomalous R-symmetry. Correspondingly, when the condition (3.6) is satisfied, there are no divergent quantum contributions to the FI parameter and classical Coulomb branch is preserved at the quantum level. The latter case is of primary importance for our studies as we are interested in the two-loop quantum contributions to the effective action on the Coulomb branch. However, in this section, for the sake of completeness we will shortly consider a model for which the condition (3.6) is not satisfied and will give a superfield derivation of the effective twisted superpotential obtained originally in [2] by component field quantum computations.

The typical example of the models for which the constraint (3.6) is violated is the supersymmetric electrodynamics with one chiral multiplet while the well-known case when this constraint is satisfied is the supersymmetric electrodynamics with two chiral multiplets carrying opposite charges under the $\mathrm{U}(1)$ gauge symmetry. The latter will be studied in section 3.3 while the former is considered just below.

\subsection{SQED with one chiral flavor}

\subsubsection{Classical action}

In this section, we consider the supersymmetric electrodynamics with one chiral multiplet carrying charge +1

$$
S=\int d^{2 \mid 4} z\left(\frac{1}{2 e^{2}} \Sigma \bar{\Sigma}-\bar{Q} e^{2 V} Q\right)-\left[\frac{i}{2} \int d^{2 \mid 2} \tilde{z}\left(t \Sigma+\frac{\mathfrak{m}}{2 e^{2}} \Sigma^{2}\right)+\text { c.c. }\right] .
$$

Obviously, in the limit $e \rightarrow \infty$ the classical action becomes scale invariant and superconformal, though this symmetry is known to be broken by quantum corrections [2].

Recall that the $\mathcal{N}=(2,2)$ vector multiplet contains a complex scalar $\sigma$ associated with the lowest component of the superfield $\Sigma$

$$
\sigma \equiv \Sigma \mid
$$

where the bar-projection means vanishing $\theta$-variables. Denoting the scalar fields in the chiral multiplet by

$$
\varphi \equiv Q|, \quad \bar{\varphi} \equiv \bar{Q}|,
$$

it is not hard to find the scalar potential which appears after elimination of auxiliary fields

$$
\mathscr{V}=\frac{e^{2}}{2}\left(\varphi \bar{\varphi}-t-\frac{\mathfrak{m}}{e^{2}} \operatorname{Re} \sigma\right)^{2}+\varphi \bar{\varphi} \sigma \bar{\sigma} .
$$


The Coulomb branch is parametrized by the vev of the scalar field $\sigma$ in the vector multiplet while the scalars from the chiral multiplet must have vanishing vevs

$$
\text { Coulomb branch: } \quad\langle\sigma\rangle=\text { const }, \quad\langle\varphi\rangle=0 .
$$

The vanishing of the scalar potential (3.10) for such values of scalars is possible only for special value of the FI parameter

$$
t=-\frac{\mathfrak{m}}{e^{2}}\langle\operatorname{Re} \sigma\rangle
$$

In this case the chiral multiplet acquires real mass proportional to the vev of $\sigma$ while the vector multiplet ('photon') has a small mass $\mathfrak{m}$. Naively, one could study the effective action for the vector multiplet which appears by integrating out the massive chiral multiplet. However, the constraint (3.12) appears to be ruined by one-loop quantum corrections and the Coulomb branch is lifted at the quantum level [3]. Although this scenario is well-known, we will demonstrate it explicitly by computing one-loop effective action in the model (3.7). The details of these computations will be of use in subsequent sections.

In general, the effective action for the vector multiplet $V$ may have odd and even parts with respect to the reflection $V \rightarrow-V$,

$$
\Gamma[V]=\Gamma_{\text {odd }}[V]+\Gamma_{\text {even }}[V],
$$

where

$$
\Gamma_{\text {odd }}[-V]=-\Gamma_{\text {odd }}[V], \quad \Gamma_{\text {even }}[-V]=\Gamma_{\text {even }}[V] .
$$

Treatment of these parts in the effective action requires slightly different computational methods. Therefore, we will consider them separately.

\subsubsection{Even part of the one-loop effective action}

Let $H$ be the operator which appears in the matrix of second variational derivatives of $S$ with respect to the chiral superfields,

$$
H=\left(\begin{array}{cc}
0 & \frac{1}{4} \bar{\nabla}^{2} \\
\frac{1}{4} \nabla^{2} & 0
\end{array}\right) .
$$

The even part of the one-loop effective action $\Gamma$ may be found by evaluating trace of logarithm of square of this operator

$$
\Gamma_{\text {even }}=\frac{i}{4} \operatorname{Tr} \ln H^{2}=\frac{i}{4} \operatorname{Tr} \ln \square_{+}+\text {c.c. }
$$

Here we have taken into account the definition (2.46) of the chiral covariant d'Alembertian in terms of covariant spinor derivatives. Associated with this operator is the Green function $G_{+}\left(z, z^{\prime}\right)$ defined in (2.48a) and the corresponding heat kernel $K_{+}\left(z, z^{\prime} \mid s\right)$, see eq. (2.50). Thus, for the effective action (3.16) we have the following proper-time representation

$$
\Gamma_{\text {even }}=-\frac{i}{4} \int_{0}^{\infty} \frac{d s}{s} \operatorname{Tr}_{+} K_{+}(s)+\text { c.c. }
$$


where $\operatorname{Tr}_{+} K_{+}(s)$ means the heat kernel $K_{+}\left(z, z^{\prime} \mid s\right)$ at coincident superspace points, $z^{\prime}=z$, and integrated over the chiral subspace

$$
\operatorname{Tr}_{+} K_{+}(s)=\int d^{2 \mid 2} z K_{+}(z, z \mid s) .
$$

This reduces the problem of computation of the even part of the effective action to evaluating the limit of coincident superspace points for the heat kernel, $\lim _{z^{\prime} \rightarrow z} K_{+}\left(z, z^{\prime} \mid s\right)$.

Recall that we consider the low-energy effective action for the on-shell, constant vector multiplet background specified by the constraints (2.34) and (2.35). For this background, the heat kernel $K_{+}$was found in the form (2.57). This formula involves different $s$-dependent objets defined in (2.39) and (2.40). For the one-loop effective action we need the values of these objets at coincident superspace points when all components of the superspace interval vanish, $\zeta^{A} \rightarrow 0$. In particular, simple calculations yield

$$
\left.\zeta^{2}(s)\right|_{\zeta^{A}=0}=s^{2} W^{2} \frac{\sinh ^{2} \frac{s f}{2}}{(s f / 2)^{2}}
$$

where $f$ is the component of the superfield strength tensor, $F_{m n}=\varepsilon_{m n} f$, which can be regarded as a constant for the considered background. It is important to note that the formula (3.19) contains $W^{2}$ that prevents any other contributions from the other $s$ depended objets in (2.57). Thus, this kernel acquires simple form at coincident superspace points

$$
K_{+}(z, z \mid s)=\frac{1}{4 \pi} s W^{2} e^{-i s \Sigma \bar{\Sigma}} \frac{\tanh (s f / 2)}{s f / 2} .
$$

Substituting this expression into (3.17) we find the even part of the one-loop effective action

$$
\Gamma_{\text {even }}=-\frac{i}{16 \pi} \int d^{2 \mid 2} z \int_{0}^{\infty} d s W^{2} e^{-i s \Sigma \bar{\Sigma}} \frac{\tanh (s f / 2)}{s f / 2}+c . c .
$$

It is instructive to rewrite the functional (3.21) in the full superspace

$$
\begin{aligned}
\Gamma_{\text {even }}= & \frac{1}{8 \pi} \int d^{2 \mid 4} z \ln \Sigma \ln \bar{\Sigma} \\
& +\frac{i}{8 \pi} \int d^{2 \mid 4} z \int_{0}^{\infty} d s e^{-i s \Sigma \bar{\Sigma}} \frac{W^{2} \bar{W}^{2}}{f^{2}}\left(\frac{\tanh (s f / 2)}{s f / 2}-1\right) .
\end{aligned}
$$

The term in the first line here specifies the effective Kähler potential for the twisted chiral superfield $\Sigma$. The term in the last line in (3.22) takes into account all higher-derivative corrections with respect to the gauge superfield which can be considered as the EulerHeisenberg effective action.

We point out that the effective action (3.22) was found for the first time in [2] using component filed one-loop computations and in [38] by means of superfield methods. Here we just gave a derivation of this effective action by taking advantage of the superfield heat kernel technique. This result will be useful in the study of low-energy effective action in the model with two chiral flavors which will be considered in section 3.3. 


\subsubsection{Odd part of the one-loop effective action}

The odd part of the effective action cannot be found upon squaring of the operator (3.15). Instead, to catch up the odd contributions we have to consider the general variation of the effective action with respect to the vector multiplet

$$
\delta \Gamma=\int d^{2 \mid 4} z \delta V\langle J\rangle,
$$

where $\langle J\rangle$ is the effective current. In the one-loop approximation, this effective current receives contributions only from the chiral superfield propagator in the vector multiplet background

$$
\langle J\rangle=-2\left\langle Q e^{2 V} \bar{Q}\right\rangle=2 i G_{+-}(z, z),
$$

where $G_{+-}\left(z, z^{\prime}\right)$ is defined in eq. (2.60a), with the mass parameter set to zero, $m=0$. It is useful also to represent this effective current via the heat kernel $K_{+-}$using (2.61a)

$$
\langle J\rangle=2 \int_{0}^{\infty} d s K_{+-}(z, z \mid s) .
$$

Thus, the computation of (3.23) is reduced to finding the trace of the heat kernel $K_{+-}\left(z, z^{\prime} \mid s\right)$.

The problem of evaluating the trace of the heat kernel $K_{+-}\left(z, z^{\prime} \mid s\right)$ is rather technically involved. However, to find the odd part of the effective action we don't actually need to now the full expression for $K_{+-}(z, z \mid s)$. Indeed, the full expression for $K_{+_{-}}(z, z \mid s)$ contains different terms which are responsible both for odd and even parts of the effective action. Since the even part of the effective action has been fully studied in the previous subsection, here we have to focus only on possible contributions to $\Gamma_{\text {odd }}$ from $K_{+-}(z, z \mid s)$. For this goal it is sufficient to approximate $K_{+-}(z, z \mid s)$ by the terms with no derivatives of $\Sigma$,

$$
K_{+-}(z, z \mid s) \approx-\frac{1}{4 \pi s} e^{-i s \Sigma \bar{\Sigma}} .
$$

Substituting (3.26) into (3.25) we have UV-divergent integral over the proper time $s$. Introducing a small regularization parameter $\epsilon$ this integral may be evaluated

$$
\left\langle J_{\epsilon}\right\rangle=-\frac{1}{2 \pi}\left(\frac{1}{\epsilon}-\gamma\right)+\frac{1}{2 \pi} \ln (\Sigma \bar{\Sigma})+O(\epsilon),
$$

where $\gamma$ is the Euler-Mascheroni constant. Thus, we see that the odd part of the effective action is the sum of divergent and finite contributions

$$
\Gamma_{\text {odd }}=\Gamma_{\text {div }}+\Gamma_{\text {fin }} .
$$

The divergent part of the effective action can be immediately read off from the first term in (3.27)

$$
\Gamma_{\mathrm{div}}=-\frac{1}{2 \pi \epsilon} \int d^{2 \mid 4} z V=\frac{i}{8 \pi \epsilon} \int d^{2 \mid 2} \tilde{z} \Sigma+\text { c.c. }
$$

This expression, being added to the classical action (3.7), leads to infinite renormalization of the FI parameter

$$
t \rightarrow t^{\prime}=t-\frac{1}{4 \pi \epsilon} .
$$


This means that even if we switch off the FI parameter classically, it is always generated by one-loop divergent tadpole diagram. This is the origin of lifting of the classical Coulomb branch by quantum corrections advocated in [3].

Substituting (3.27) into (3.23) we get the variation of finite terms in the odd part of the effective action

$$
\delta \Gamma_{\text {fin }}=\frac{1}{2 \pi} \int d^{2 \mid 4} z \delta V \ln (\Sigma \bar{\Sigma}) .
$$

Integrating this variation we uncover the effective twisted potential for $\Sigma$

$$
\Gamma_{\text {fin }}=-\frac{i}{4 \pi} \int d^{2 \mid 2} \tilde{z} \Sigma(\ln \Sigma-1)+\text { c.c. }
$$

This effective twisted potential was found originally in [2] using component field quantum computations. Here we reproduced the same result using the method of covariant perturbative computations in the $\mathcal{N}=(2,2)$ superspace.

The above results can be readily generalized to the case of electrodynamics with $N$ chiral flavors $Q_{i}, i=1, \ldots, N$, with charges $q_{i}$, see eq. (3.5). For the odd part of the effective action we have the following modification of formulas (3.29) and (3.32):

$$
\begin{aligned}
\Gamma_{\mathrm{div}} & =\frac{i}{8 \pi \epsilon} \sum_{i=1}^{N} q_{i} \int d^{2 \mid 2} \tilde{z} \Sigma+\text { c.c. } \\
\Gamma_{\mathrm{fin}} & =-\frac{i}{4 \pi} \sum_{i=1}^{N} q_{i} \ln q_{i} \int d^{2 \mid 2} \tilde{z} \Sigma-\frac{i}{4 \pi} \sum_{i=1}^{N} q_{i} \int d^{2 \mid 2} \tilde{z} \Sigma(\ln \Sigma-1)+\text { c.c. }
\end{aligned}
$$

The equation (3.33) implies that there is no infinite renormalization of the FI parameter when the condition (3.6) is satisfied. This is the necessary condition for existence of the Coulomb branch. This condition is also sufficient for vanishing of the effective twisted potential in (3.34). However, even when the condition (3.6) is satisfied, the first term in the right-hand side of (3.34) remains non-vanishing and yields a finite shift of the complex FI parameter

$$
t \rightarrow t^{\prime}=t+\frac{1}{2 \pi} \sum_{i=1}^{N} q_{i} \ln q_{i} .
$$

The main effect of this finite quantum contribution is the shift of the imaginary part $\theta$ of the complex FI parameter (3.4). To compensate this shift, one has to add the corresponding value to the classical FI parameter

$$
t=-\frac{\mathfrak{m}}{e^{2}}\langle\operatorname{Re} \sigma\rangle-\frac{1}{2 \pi} \sum_{i=1}^{N} q_{i} \ln q_{i} .
$$

This is the sufficient condition of existence of the Coulomb branch on the quantum level [5].

To summarize, we have shown that the FI parameter in the model (3.7) receives infinite one-loop quantum contributions (3.30) which lift the classical Coulomb branch. Such infinite contributions may cancel among each other in the generalization of the model (3.7) which involves $N$ charged chiral flavors (3.5). This happens when all charges of chiral 
multiplets sum to zero (3.6). However, there is still a finite shift of the imaginary part of the FI parameter as in (3.35). Therefore, quantum Coulomb branch exists when the classical FI parameter is tuned to a special value (3.36). Since we are interested in the effective action in the Coulomb branch, in subsequent sections we will always assume that the condition (3.36) is satisfied.

We stress that all results of this subsection are not new; they are well-known owing to $[2,3,5]$. Here we just summarized them for the sake of completeness of our consideration.

\subsection{SQED with two chiral flavors}

\subsubsection{Classical action and background field setup}

Let us consider supersymmetric electrodynamics with two chiral multiplets $Q_{+}$and $Q_{-}$ carrying charges \pm 1 , respectively,

$$
\begin{aligned}
S & =S_{\text {gauge }}[V]+S_{\text {mat }}[Q, V] \\
S_{\text {gauge }}[V] & =\frac{1}{2 e^{2}} \int d^{2 \mid 4} z \Sigma \bar{\Sigma}-\left[\frac{i}{2} \int d^{2 \mid 2} \tilde{z}\left(t \Sigma+\frac{\mathfrak{m}}{2 e^{2}} \Sigma^{2}\right)+\text { c.c. }\right] \\
S_{\text {mat }}[Q, V] & =-\int d^{2 \mid 4} z\left(\bar{Q}_{+} e^{2 V} Q_{+}+\bar{Q}_{-} e^{-2 V} Q_{-}\right)-\left(m \int d^{2 \mid 2} z Q_{+} Q_{-}+\text {c.c. }\right),
\end{aligned}
$$

where $m$ is the mass of the chiral multiplet while $\mathfrak{m}$ is the vector multiplet mass. The latter is assumed to be small as compared to the former,

$$
\mathfrak{m}^{2} \ll m^{2}+\langle\Sigma \bar{\Sigma}\rangle
$$

In this regime, we can study the effective action for the light field $\Sigma$ which appears upon integrating out the heavy chirals $Q_{ \pm}$. In what follows, without loss of generality we will assume that both $m$ and $\mathfrak{m}$ are real, though, in general, they may be complex.

Let $\varphi_{ \pm}$be scalar fields in the chiral multiplets

$$
\varphi_{ \pm}=Q_{ \pm}\left|, \quad \bar{\varphi}_{ \pm}=\bar{Q}_{ \pm}\right| .
$$

After elimination of auxiliary fields, one can readily find the scalar potential

$$
\mathscr{V}=\frac{e^{2}}{2}\left(\varphi_{+} \bar{\varphi}_{+}-\varphi_{-} \bar{\varphi}_{-}-t-\frac{\mathfrak{m}}{e^{2}} \operatorname{Re} \sigma\right)^{2}+\left(\sigma \bar{\sigma}+m^{2}\right)\left(\varphi_{+} \bar{\varphi}_{+}+\varphi_{-} \bar{\varphi}_{-}\right) .
$$

Similarly as in the model (3.7), the classical Coulomb branch (3.11) is possible at the special value of the FI parameter (3.12). However, as is explained in the previous section, the imaginary part of the FI parameter receives finite one-loop contributions as in eq. (3.35). To compensate this contribution, we have to set up the corresponding value to the classical FI parameter

$$
t=\frac{i}{2}-\frac{\mathfrak{m}}{e^{2}}\langle\operatorname{Re} \sigma\rangle
$$

This allows us to study the low-energy effective action for the light vector multiplet which appears by integrating out heavy chiral multiplets beyond one-loop order. 
In the framework of the background field method, we split the gauge superfield $V$ into background $V$ and quantum $v$ parts ${ }^{2}$

$$
V \rightarrow V+e v .
$$

Upon this splitting, the actions (3.37a) and (3.37c) decompose as

$$
\begin{aligned}
S_{\text {gauge }}[V] \rightarrow & S_{\text {gauge }}[V]+\frac{1}{e} \int d^{2 \mid 4} z v\left(i D^{\alpha} W_{\alpha}+2 \mathfrak{m} \Sigma+2 e^{2} t\right) \\
& +\int d^{2 \mid 4} z v\left(\frac{1}{8} D^{\alpha} \bar{D}^{2} D_{\alpha}+i \mathfrak{m} D^{\alpha} \bar{D}_{\alpha}\right) v, \\
S_{\text {mat }}[Q, V] \rightarrow & S_{\text {mat }}[\mathcal{Q}, v],
\end{aligned}
$$

where $\mathcal{Q}_{ \pm}$and $\overline{\mathcal{Q}}_{ \pm}$are covariantly (anti)chiral superfields with respect to the background gauge superfield

$$
\overline{\mathcal{Q}}_{+}=\bar{Q}_{+} e^{2 V}, \quad \mathcal{Q}_{+}=Q_{+}, \quad \overline{\mathcal{Q}}_{-}=\bar{Q}_{-} e^{-2 V}, \quad \mathcal{Q}_{-}=Q_{-} .
$$

The operators $D^{\alpha} \bar{D}^{2} D_{\alpha}$ and $D^{\alpha} \bar{D}_{\alpha}$ in (3.43a) are degenerate and require gauge fixing. The gauge fixing is implemented by adding to the action (3.43a) the following term

$$
S_{\mathrm{gf}}=\int d^{2 \mid 4} z v\left[-\frac{1}{16}\left\{D^{2}, \bar{D}^{2}\right\}+\frac{i \mathfrak{m}}{4}\left(D^{2}+\bar{D}^{2}\right)\right] v .
$$

This gauge-fixing action appears upon inserting the standard delta-functions $\delta\left[f-i \bar{D}^{2} v\right] \times$ $\delta\left[\bar{f}-i D^{2} v\right]$ into the functional integral over $\mathcal{D} v$ and averaging them with appropriate weight (see [39] for details of this procedure in the three-dimensional case). After gauge fixing, we get the action for 'quantum' fields

$$
\begin{aligned}
S_{\text {quant }} & =S_{2}+S_{\text {int }} \\
S_{2} & =-\int d^{2 \mid 4} z\left[v(\square-H) v+\overline{\mathcal{Q}}_{+} \mathcal{Q}_{+}+\overline{\mathcal{Q}}_{-} \mathcal{Q}_{-}\right]-\left(m \int d^{2 \mid 2} z \mathcal{Q}_{+} \mathcal{Q}_{-}\right) \\
S_{\text {int }} & =-2 \int d^{2 \mid 4} z\left[e\left(\overline{\mathcal{Q}}_{+} \mathcal{Q}_{+}-\overline{\mathcal{Q}}_{-} \mathcal{Q}_{-}\right) v+e^{2}\left(\overline{\mathcal{Q}}_{+} \mathcal{Q}_{+}+\overline{\mathcal{Q}}_{-} \mathcal{Q}_{-}\right) v^{2}\right]+O\left(e^{3}\right)
\end{aligned}
$$

where

$$
H=\frac{i \mathfrak{m}}{4}\left(2 D^{\alpha} \bar{D}_{\alpha}+D^{2}+\bar{D}^{2}\right) .
$$

This operator obeys the important property

$$
H^{2}=-\mathfrak{m}^{2} \square .
$$

This identity allows us to represent the propagator for the superfield $v$ in the form

$$
\begin{aligned}
2 i\left\langle v(z) v\left(z^{\prime}\right)\right\rangle & \equiv G_{v}\left(z, z^{\prime}\right)=\frac{1}{\square-H} \delta^{2 \mid 4}\left(z-z^{\prime}\right) \\
& =-i \int_{0}^{\infty} d s\left[e^{-i s \mathfrak{m}^{2}}+\frac{H}{\mathfrak{m}^{2}}\left(1-e^{-i s \mathfrak{m}^{2}}\right)\right] K_{0}\left(z, z^{\prime} \mid s\right),
\end{aligned}
$$

\footnotetext{
${ }^{2}$ The background gauge superfield is denoted by the same letter as the original superfield $V$. This should not lead to any confusions as the original unsplit gauge superfield does not show up after the backgroundquantum splitting.
} 
where

$$
K_{0}\left(z, z^{\prime} \mid s\right)=-\frac{1}{4 \pi s} e^{-\frac{i \rho^{2}}{4 s}} \zeta^{2} \bar{\zeta}^{2} .
$$

Here $\rho^{m}, \zeta^{\alpha}$ and $\bar{\zeta}^{\alpha}$ are the components of the supersymmetric interval (2.21).

In addition to the photon propagator (3.49), the action (3.46b) yields the propagators of chiral superfields

$$
\begin{aligned}
& i\left\langle\mathcal{Q}_{+}(z) \mathcal{Q}_{-}\left(z^{\prime}\right)\right\rangle=-m G_{+}\left(z, z^{\prime}\right), \\
& i\left\langle\mathcal{Q}_{+}(z) \overline{\mathcal{Q}}_{+}\left(z^{\prime}\right)\right\rangle=G_{+-}\left(z, z^{\prime}\right)=G_{-+}\left(z^{\prime}, z\right), \\
& i\left\langle\overline{\mathcal{Q}}_{-}(z) \mathcal{Q}_{-}\left(z^{\prime}\right)\right\rangle=G_{-+}\left(z, z^{\prime}\right),
\end{aligned}
$$

where Green's functions $G_{+}$and $G_{+-}$are defined by the equations (2.48a) and (2.60a), respectively.

Using the form of cubic and quartic interaction vertices for quantum fields in (3.46c), we deduce the formal decomposition of the effective action up to two-loop order

$$
\begin{aligned}
\Gamma & =\Gamma^{(1)}+\Gamma^{(2)} \\
\Gamma^{(1)} & =i \operatorname{Tr} \ln \left(\square_{+}+m^{2}\right) \\
\Gamma^{(2)} & =-2 e^{2} \int d^{2 \mid 4} z d^{2 \mid 4} z^{\prime}\left[G_{+-}\left(z, z^{\prime}\right) G_{+-}\left(z^{\prime}, z\right)+m^{2} G_{+}\left(z, z^{\prime}\right) G_{-}\left(z, z^{\prime}\right)\right] G_{v}\left(z, z^{\prime}\right) .
\end{aligned}
$$

Here $\Gamma^{(1)}$ is the one-loop effective action while $\Gamma^{(2)}$ takes into account two-loop quantum corrections. These quantum contributions will be calculated separately in the subsequent sections.

\subsubsection{One-loop effective action}

The computation of the one-loop effective action in the model (3.37) is very similar to the one for SQED with one chiral flavor considered in section 3.2. However, it has some important features.

First of all, the effective action in the model (3.37) possesses no odd part with respect to the reflection $V \rightarrow-V$. As is demonstrated in section 3.2.3, the odd contributions to the effective action cancel against each other in the model where the charges of flavors sum to zero, (3.6). Thus, we have to focus only on the even part of the one-loop effective action.

The computation of the even part of the effective action goes along the same lines as in section 3.2.2. Following these steps, one arrives at the expression (3.21), with two simple modifications: (i) The result (3.21) should be multiplied by 2 as we have contributions from two chiral flavors now; (ii) the mass parameter $m$ should be inserted,

$$
\Gamma^{(1)}=-\frac{i}{8 \pi} \int d^{2 \mid 2} z \int_{0}^{\infty} d s W^{2} e^{-i s\left(\Sigma \bar{\Sigma}+m^{2}\right)} \frac{\tanh (s f / 2)}{s f / 2}+c . c .
$$

It is an instructive exercise to rewrite the functional (3.53) in the full superspace. We give the details of this procedure for the chiral part of (3.53); the antichiral part can be analyzed in the same way. 
At the first step, we identically rewrite the chiral part of (3.53) as the sum of two terms

$$
\begin{aligned}
\Delta \equiv & -\frac{i}{8 \pi} \int d^{2 \mid 2} z \int_{0}^{\infty} d s W^{2} e^{-i s\left(\Sigma \bar{\Sigma}+m^{2}\right)} \frac{\tanh (s f / 2)}{s f / 2} \\
= & -\frac{i}{8 \pi} \int d^{2 \mid 2} z \int_{0}^{\infty} d s W^{2} e^{-i s\left(\Sigma \bar{\Sigma}+m^{2}\right)} \\
& -\frac{i}{8 \pi} \int d^{2 \mid 2} z \int_{0}^{\infty} d s \frac{W^{2} \bar{D}^{2} \bar{W}^{2}}{4 f^{2}} e^{-i s\left(\Sigma \bar{\Sigma}+m^{2}\right)}\left(\frac{\tanh (s f / 2)}{s f / 2}-1\right),
\end{aligned}
$$

where, in the last line, we have inserted the unity, $1=\frac{1}{4 f^{2}} \bar{D}^{2} \bar{W}^{2}$. In this identity, the operator $\bar{D}^{2}$ can be used to restore the full superspace measure due to (A.8). Then, after evaluation of the proper-time integral in the second line of (3.54), we have

$$
\begin{aligned}
\Delta= & -\frac{1}{8 \pi} \int d^{2 \mid 2} z \frac{W^{2}}{\Sigma \bar{\Sigma}+m^{2}} \\
& +\frac{i}{8 \pi} \int d^{2 \mid 4} z \int_{0}^{\infty} d s e^{-i s\left(\Sigma \bar{\Sigma}+m^{2}\right)} \frac{W^{2} \bar{W}^{2}}{f^{2}}\left(\frac{\tanh (s f / 2)}{s f / 2}-1\right) .
\end{aligned}
$$

Next, we have to restore the full superspace measure in the first line of (3.55) using the operators $\bar{D}_{\alpha}$ from $W_{\alpha}=\bar{D}_{\alpha} G=i\left(\gamma^{3}\right)_{\alpha}^{\beta} \bar{D}_{\beta} H$, see (2.5). Making use of properties of the superfield strengths (2.4) and (2.5), one can prove the identity

$$
\int d^{2 \mid 4} z \mathcal{F}(X)=-\int d^{2 \mid 2} z W^{2}\left[\left(X-m^{2}\right) \mathcal{F}^{\prime \prime}(X)+\mathcal{F}^{\prime}(X)\right]
$$

for some function $\mathcal{F}(X)$ and $X \equiv \Sigma \bar{\Sigma}+m^{2}$. Comparing the right-hand side of (3.56) with the first line of (3.55), one finds the following differential equation for this function

$$
\left(X-m^{2}\right) \mathcal{F}^{\prime \prime}(X)+\mathcal{F}^{\prime}(X)=\frac{1}{X}
$$

with the general solution

$$
\mathcal{F}(X)=c_{1}+c_{2} \ln \left(X-m^{2}\right)+\frac{1}{2} \ln ^{2} \frac{X-m^{2}}{m^{2}}+\operatorname{Li}_{2}\left(-\frac{m^{2}}{X-m^{2}}\right),
$$

where $c_{1}$ and $c_{2}$ are arbitrary constants of integration. The terms with these constants drop out upon integration over the full superspace owing to the properties (2.14) and (2.15). The remaining two terms in (3.58) allow us to get the full-superspace representation for the first term in (3.55)

$$
-\frac{1}{8 \pi} \int d^{2 \mid 2} z \frac{W^{2}}{\Sigma \bar{\Sigma}+m^{2}}=\frac{1}{8 \pi} \int d^{2 \mid 4} z\left[\ln \Sigma \ln \bar{\Sigma}+\operatorname{Li}_{2}\left(-\frac{m^{2}}{\Sigma \bar{\Sigma}}\right)\right] .
$$

Note that the last term in (3.59) vanishes in the limit $m=0$ owing to the identity $\mathrm{Li}_{2}(0)=0$. In this limit, the expression (3.59) coincides with the non-holomorphic potential in (3.22). 
Recall that we considered here the chiral part of (3.53). It can be shown that the antichiral part gives the same contribution as (3.55), so that $\Gamma^{(1)}=2 \Delta$. Thus, substituting (3.59) into (3.55), we end up with the representation for the one-loop effective action in the full superspace

$$
\begin{aligned}
\Gamma^{(1)}= & \frac{1}{4 \pi} \int d^{2 \mid 4} z\left[\ln \Sigma \ln \bar{\Sigma}+\operatorname{Li}_{2}\left(-\frac{m^{2}}{\Sigma \bar{\Sigma}}\right)\right] \\
& +\frac{i}{4 \pi} \int d^{2 \mid 4} z \int_{0}^{\infty} d s e^{-i s\left(\Sigma \bar{\Sigma}+m^{2}\right)} \frac{W^{2} \bar{W}^{2}}{f^{2}}\left(\frac{\tanh (s f / 2)}{s f / 2}-1\right) .
\end{aligned}
$$

The term in the first line here can be interpreted as the one-loop quantum correction to the effective Kähler potential for the twisted chiral superfield $\Sigma$

$$
K^{(1)}=\frac{1}{4 \pi} \ln \Sigma \ln \bar{\Sigma}+\frac{1}{4 \pi} \operatorname{Li}_{2}\left(-\frac{m^{2}}{\Sigma \bar{\Sigma}}\right) .
$$

The second line in (3.60) is responsible for higher-derivative corrections in the one-loop Euler-Heisenberg-type action.

\subsubsection{Two-loop effective Kähler potential}

In principle, starting from (3.52c) it is possible to determine two-loop quantum corrections to the Euler-Heisenberg-type action. ${ }^{3}$ However, the form of the resulting expression appears not very illuminating as it involves numerous proper-time integrations and may have very limited applications. Therefore, in this section we restrict ourself to studying two-loop quantum corrections only to the effective Kähler potential for the twisted chiral superfield $\Sigma$. To this aim, it is sufficient to consider the gauge superfield background constrained by

$$
W_{\alpha}=0, \quad \bar{W}_{\alpha}=0,
$$

while superfields $\Sigma$ and $\bar{\Sigma}$ are constant and non-vanishing. In this approximation, the heat kernels (2.57) and (2.68) reduce to

$$
\begin{aligned}
K_{+}\left(z, z^{\prime} \mid s\right) & \approx \frac{1}{4 \pi s} e^{-i s \Sigma \bar{\Sigma}} e^{-i \frac{\rho^{2}}{4 s}} \zeta^{2} I\left(z, z^{\prime}\right), \\
K_{+-}\left(z, z^{\prime} \mid s\right) & \approx-\frac{1}{4 \pi s} e^{-i s \Sigma \bar{\Sigma}} e^{-i \frac{\rho^{2}}{4 s}} I\left(z, z^{\prime}\right) .
\end{aligned}
$$

The two-loop effective action (3.52c) is given by the sum of two terms, which we denote by $\Gamma_{\mathrm{A}}$ and $\Gamma_{\mathrm{B}}$, respectively,

$$
\begin{aligned}
\Gamma^{(2)} & =\Gamma_{\mathrm{A}}+\Gamma_{\mathrm{B}}, \\
\Gamma_{\mathrm{A}} & =-2 e^{2} \int d^{2 \mid 4} z d^{2 \mid 4} z^{\prime} G_{+-}\left(z, z^{\prime}\right) G_{+-}\left(z^{\prime}, z\right) G_{v}\left(z, z^{\prime}\right), \\
\Gamma_{\mathrm{B}} & =-2 e^{2} m^{2} \int d^{2 \mid 4} z d^{2 \mid 4} z^{\prime} G_{+}\left(z, z^{\prime}\right) G_{-}\left(z, z^{\prime}\right) G_{v}\left(z, z^{\prime}\right) .
\end{aligned}
$$

\footnotetext{
${ }^{3}$ Note that in the non-supersymmetric two-dimensional electrodynamics the Euler-Heisenberg effective action was studied in [40] up to the two-loop order. We point out that beyond one loop this effective action in the supersymmetric QED cannot be found by simple composition of non-supersymmetric results in scalar and spinor electrodynamics.
} 


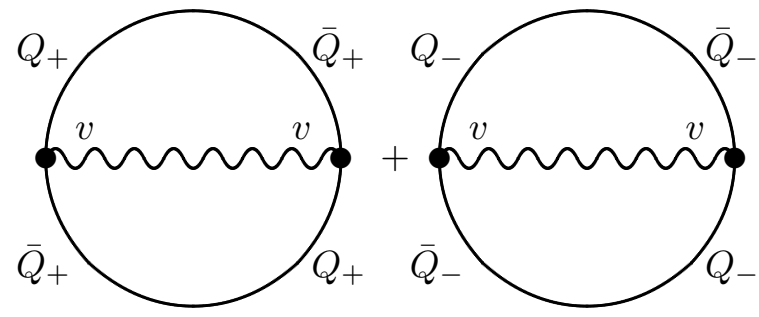

Type A

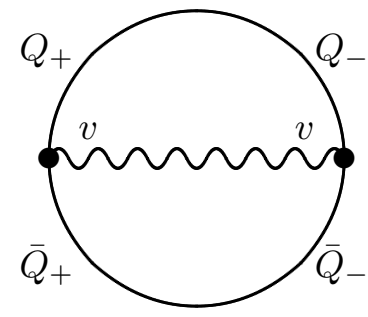

Type B

Figure 1. Two-loop supergraphs in $\mathcal{N}=(2,2)$ supersymmetric electrodynamics.

These two terms correspond to the Feynman graphs of types A and B in figure 1. They have slightly different structure and need to be considered separately. Note that the twoloop graph of the topology ' 8 ' vanishes identically and, thus, does not show up in (3.65).

It is possible to show that the part of effective action (3.65c) does not contribute to the effective Kähler potential. In this formula, we express Green's functions via the corresponding heat kernels (2.50) and (3.49)

$$
\begin{aligned}
\Gamma_{\mathrm{B}}= & -2 i e^{2} m^{2} \int d^{2 \mid 4} z d^{2 \mid 4} z^{\prime} \int_{0}^{\infty} d s d t d u e^{-i(s+t) m^{2}} K_{+}\left(z, z^{\prime} \mid s\right) K_{-}\left(z, z^{\prime} \mid t\right) \\
& \times\left[e^{-i u \mathfrak{m}^{2}}+\frac{H}{\mathfrak{m}^{2}}\left(1-e^{-i u \mathfrak{m}^{2}}\right)\right] K_{0}\left(z, z^{\prime} \mid u\right) .
\end{aligned}
$$

The operator $H$ in the last line contains the covariant spinor derivatives (see eq. (3.47)), which can be integrated by parts

$$
\begin{aligned}
\Gamma_{\mathrm{B}}= & 2 i e^{2} m^{2} \int d^{2 \mid 4} z d^{2} \rho \int_{0}^{\infty} \frac{d s d t d u}{4 \pi u} e^{-i(s+t) m^{2}} e^{-i \frac{\rho^{2}}{4 u}} \\
& \times\left.\left[e^{-i u \mathfrak{m}^{2}}+\frac{H}{\mathfrak{m}^{2}}\left(1-e^{-i u \mathfrak{m}^{2}}\right)\right] K_{+}\left(z, z^{\prime} \mid s\right) K_{-}\left(z, z^{\prime} \mid t\right)\right|_{\zeta \rightarrow 0},
\end{aligned}
$$

where we have taken into account the explicit form of the heat kernel (3.50). The last line in (3.67) contains the terms of the following three types:

$$
\begin{aligned}
& \left.K_{+}\left(z, z^{\prime} \mid s\right) K_{-}\left(z, z^{\prime} \mid t\right)\right|_{\zeta \rightarrow 0}, \\
& \left.\nabla^{2} K_{+}\left(z, z^{\prime} \mid s\right) K_{-}\left(z, z^{\prime} \mid t\right)\right|_{\zeta \rightarrow 0},\left.\quad K_{+}\left(z, z^{\prime} \mid s\right) \bar{\nabla}^{2} K_{-}\left(z, z^{\prime} \mid t\right)\right|_{\zeta \rightarrow 0}, \\
& \left.\nabla^{\alpha} K_{+}\left(z, z^{\prime} \mid s\right) \bar{\nabla}_{\alpha} K_{-}\left(z, z^{\prime} \mid t\right)\right|_{\zeta \rightarrow 0} .
\end{aligned}
$$

The terms involving (3.68a) cannot contribute to the effective Kähler potential since the expression (3.20) vanishes in the approximation (3.62). For the same reason there are no contributions from the terms (3.68b). In a similar way it is easy to argue that the expressions (3.68c) cannot contribute to the effective Kähler potential owing to the properties

$$
\left.\nabla_{\alpha} K_{+}\left(z, z^{\prime} \mid s\right)\right|_{\zeta \rightarrow 0} \propto W_{\alpha},\left.\quad \bar{\nabla}_{\alpha} K_{-}\left(z, z^{\prime} \mid t\right)\right|_{\zeta \rightarrow 0} \propto \bar{W}_{\alpha}
$$


These properties follow from the explicit form of the heat kernels (2.57) and (2.58). Thus, in the approximation (3.62)

$$
\Gamma_{\mathrm{B}}=0 \text {. }
$$

Now let us consider the contributions to the effective Kähler potential from the part of the effective action (3.65b). Making use of the identities (2.61) and (3.49), this effective action can be cast to the form

$$
\begin{aligned}
\Gamma_{\mathrm{A}}= & 2 i e^{2} \int d^{2 \mid 4} z d^{2 \mid 4} z^{\prime} \int_{0}^{\infty} \frac{d s d t d u}{4 \pi u} e^{-i m^{2}(s+t)} e^{-\frac{i \rho^{2}}{4 u}} \\
& \times\left.\left[e^{-i u \mathfrak{m}^{2}}+\frac{H}{\mathfrak{m}^{2}}\left(1-e^{-i u \mathfrak{m}^{2}}\right)\right] K_{+-}\left(z, z^{\prime} \mid s\right) K_{+-}\left(z^{\prime}, z \mid t\right)\right|_{\zeta \rightarrow 0} .
\end{aligned}
$$

It is easy to argue that the terms with the operator $H$ in the last line of (3.71) give no contributions to the effective Kähler potential. Indeed, covariant spinor derivatives in this operator can hit the heat kernels yielding the terms

$$
\begin{aligned}
& \left.\nabla^{2} K_{+-}\left(z, z^{\prime} \mid s\right) K_{-+}\left(z, z^{\prime} \mid t\right)\right|_{\zeta \rightarrow 0},\left.\quad K_{+-}\left(z, z^{\prime} \mid s\right) \bar{\nabla}^{2} K_{-+}\left(z, z^{\prime} \mid t\right)\right|_{\zeta \rightarrow 0}, \\
& \left.\nabla^{\alpha} K_{+-}\left(z, z^{\prime} \mid s\right) \bar{\nabla}_{\alpha} K_{-+}\left(z, z^{\prime} \mid t\right)\right|_{\zeta \rightarrow 0} .
\end{aligned}
$$

For such terms one can derive the following identities

$$
\begin{aligned}
\left.\frac{1}{4} \nabla^{2} K_{+-}\left(z, z^{\prime} \mid s\right)\right|_{\zeta \rightarrow 0} & =\left.i \frac{d}{d s} K_{-}\left(z, z^{\prime} \mid s\right)\right|_{\zeta \rightarrow 0} \propto \bar{W}^{2}, \\
\left.\frac{1}{4} \bar{\nabla}^{2} K_{-+}\left(z, z^{\prime} \mid s\right)\right|_{\zeta \rightarrow 0} & =\left.i \frac{d}{d s} K_{+}\left(z, z^{\prime} \mid s\right)\right|_{\zeta \rightarrow 0} \propto W^{2}, \\
\left.\nabla_{\alpha} K_{+-}\left(z, z^{\prime} \mid s\right)\right|_{\zeta \rightarrow 0} & \propto \bar{W}_{\alpha},\left.\quad \bar{\nabla}_{\alpha} K_{-+}\left(z, z^{\prime} \mid t\right)\right|_{\zeta \rightarrow 0} \propto W_{\alpha} .
\end{aligned}
$$

Thus, contributions from these terms to the effective action vanish in the approximation (3.62).

Non-trivial contributions to (3.71) appear only from the terms without the operator $H$

$$
\Gamma_{\mathrm{A}}=\frac{i e^{2}}{32 \pi^{3}} \int d^{2 \mid 4} z d^{2} \rho \int_{0}^{\infty} \frac{d s d t d u}{s t u} e^{-i(s+t)\left(m^{2}+\Sigma \bar{\Sigma}\right)} e^{-i u \mathfrak{m}^{2}} e^{-\frac{i \rho^{2}}{4}\left(s^{-1}+t^{-1}+u^{-1}\right)} .
$$

Here, the Gaussian integral over $d^{2} \rho$ can be easily evaluated

$$
\Gamma_{\mathrm{A}}=\frac{i e^{2}}{8 \pi^{2}} \int d^{2 \mid 4} z \int_{0}^{\infty} \frac{d s d t d u}{t u+s u+s t} e^{-i u \mathfrak{m}^{2}} e^{-i(s+t)\left(m^{2}+\Sigma \bar{\Sigma}\right)} .
$$

Finally, it is possible to perform integration over one of the parameters, say $u$, and to represent the corresponding contribution to the effective action in the form

$$
\begin{aligned}
\Gamma_{\mathrm{A}} & =\int d^{2 \mid 4} z K^{(2)}(\Sigma, \bar{\Sigma}), \\
K^{(2)}(\Sigma, \bar{\Sigma}) & =\frac{i e^{2}}{8 \pi^{2}} \int_{0}^{\infty} \frac{d s d t}{s+t} e^{-i(s+t)\left(m^{2}+\Sigma \bar{\Sigma}\right)} e^{i \frac{\mathfrak{m}^{2} s t}{s+t}} E_{1}\left(\frac{i \mathfrak{m}^{2} s t}{s+t}\right),
\end{aligned}
$$


where $E_{1}(z)$ is the exponential integral

$$
E_{1}(z)=\int_{1}^{\infty} \frac{d t}{t} e^{-t z}
$$

The expression (3.76b) represents the two-loop quantum correction to the effective Kähler potential for the twisted chiral superfield $\Sigma$. This formula involves integration over the parameters $s$ and $t$ which are hard to evaluate for generic values of masses $m$ and $\mathfrak{m}$. However, it is possible to find explicitly the leading contributions to the effective Kähler potential for small photon mass, i.e., in the regime (3.38). In this case, we can use the asymptotics of the function $E_{1}(i x)$ for small $x$,

$$
E_{1}(i x)=-\frac{i \pi}{2}-\gamma-\ln x+O(x) .
$$

The integrals over $s$ and $t$ reduce to

$$
\begin{aligned}
\int_{0}^{\infty} \frac{d s d t}{s+t} e^{-(s+t) a} & =\frac{1}{a} \\
\int_{0}^{\infty} \frac{d s d t}{s+t} e^{-(s+t) a} \ln \frac{s t}{s+t} & =-\frac{2+\gamma}{a}-\frac{1}{a} \ln a,
\end{aligned}
$$

where $a=i\left(m^{2}+\Sigma \bar{\Sigma}\right)$. This yields the simple expression for $(3.76 \mathrm{~b})$

$$
K^{(2)}(\Sigma, \bar{\Sigma})=\frac{e^{2}}{4 \pi^{2}} \frac{1}{m^{2}+\Sigma \bar{\Sigma}}+\frac{e^{2}}{8 \pi^{2}} \frac{1}{m^{2}+\Sigma \bar{\Sigma}} \ln \frac{m^{2}+\Sigma \bar{\Sigma}}{\mathfrak{m}^{2}} .
$$

This formula is a good approximation for the two-loop quantum correction to the effective Kähler potential for $\Sigma$ in the regime (3.38), i.e., when the photon possesses a small but non-vanishing mass $\mathfrak{m}$. Obviously, (3.80) is singular in the limit $\mathfrak{m} \rightarrow 0$. It emphasizes a feature of two-dimensional electrodynamics that the quantum loop diagrams with internal photon lines suffer from IR singularities unless the photon possesses a mass.

In conclusion of this section, let us consider the full effective Kähler potential $K(\Sigma, \bar{\Sigma})$ which contains the classical part $\frac{1}{2 e^{2}} \Sigma \bar{\Sigma}$ and includes both one- and two-loop quantum corrections (3.61) and (3.80)

$$
\begin{aligned}
K(\Sigma, \bar{\Sigma})= & \frac{1}{2 e^{2}} \Sigma \bar{\Sigma}+\frac{1}{4 \pi} \ln \Sigma \ln \bar{\Sigma}+\frac{1}{4 \pi} \operatorname{Li}_{2}\left(-\frac{m^{2}}{\Sigma \bar{\Sigma}}\right) \\
& +\frac{e^{2}}{4 \pi^{2}} \frac{1}{m^{2}+\Sigma \bar{\Sigma}}+\frac{e^{2}}{8 \pi^{2}} \frac{1}{m^{2}+\Sigma \bar{\Sigma}} \ln \frac{m^{2}+\Sigma \bar{\Sigma}}{\mathfrak{m}^{2}} .
\end{aligned}
$$

The corresponding sigma-model metric reads

$$
\begin{aligned}
d s^{2} & =\partial_{z} \partial_{\bar{z}} K(z, \bar{z}) d z d \bar{z} \\
& =\left(\frac{1}{2 e^{2}}+\frac{1}{4 \pi} \frac{1}{z \bar{z}+m^{2}}-\frac{e^{2}}{8 \pi^{2}} \frac{m^{2}}{\left(m^{2}+z \bar{z}\right)^{3}}+\frac{e^{2}}{8 \pi^{2}} \frac{z \bar{z}-m^{2}}{\left(m^{2}+z \bar{z}\right)^{3}} \ln \frac{m^{2}+z \bar{z}}{\mathfrak{m}^{2}}\right) d z d \bar{z} .
\end{aligned}
$$

For vanishing mass of the chiral multiplet, $m=0$, this metric acquires a simple form

$$
\left.d s^{2}\right|_{m=0}=\left(\frac{1}{2 e^{2}}+\frac{1}{4 \pi} \frac{1}{z \bar{z}}+\frac{e^{2}}{8 \pi^{2}} \frac{1}{(z \bar{z})^{2}} \ln \frac{z \bar{z}}{\mathfrak{m}^{2}}\right) d z d \bar{z} .
$$


We stress that this metric makes sense for a small but non-vanishing photon mass $\mathfrak{m}$.

The two-loop Kähler potential (3.81) and the corresponding metric (3.82) are new results obtained here by direct quantum computations in the $\mathcal{N}=(2,2)$ superspace. Though the one-loop quantum corrections to this metric were found long ago in [2], to the best of our knowledge the two-loop quantum corrections have never been presented before.

\section{Low-energy effective action in $\mathcal{N}=(4,4)$ SQED}

\subsection{Classical action and loop expansion of the effective action}

The $(4,4)$ vector multiplet may be described by the $\mathcal{N}=(2,2)$ vector multiplet $V$ and a chiral multiplet $\Phi$. The hypermultiplet is described by the pair of chiral fields $\left(Q_{+}, Q_{-}\right)$. Let us consider the following action for these multiplets

$$
\begin{aligned}
S= & S_{\text {gauge }}[V, \Phi]+S_{\text {mat }}[Q, V, \Phi] \\
S_{\text {guage }}[V, \Phi]= & \frac{1}{2 e^{2}} \int d^{2 \mid 4} z(\Sigma \bar{\Sigma}-\Phi \bar{\Phi}) \\
& -\left[\frac{i t}{2} \int d^{2 \mid 2} \tilde{z} \Sigma+\frac{i \mathfrak{m}}{4 e^{2}}\left(\int d^{2 \mid 2} z \Phi^{2}-\int d^{2 \mid 2} \tilde{z} \Sigma^{2}\right)+\text { c.c. }\right] \\
S_{\text {mat }}[Q, V, \Phi]= & -\int d^{2 \mid 4} z\left(\bar{Q}_{+} e^{2 V} Q_{+}+\bar{Q}_{-} e^{-2 V} Q_{-}\right)-\left(\int d^{2 \mid 2} z Q_{+} \Phi Q_{-}+\text {c.c. }\right) .
\end{aligned}
$$

For $\mathfrak{m}=0$ this action is invariant under 'hidden' $(2,2)$ supersymmetry with anticommuting parameters $\epsilon_{\alpha}$ and $\bar{\epsilon}_{\alpha}$

$$
\begin{array}{rlrl}
\delta V & =\frac{1}{2}\left(\bar{\epsilon}^{\alpha} \bar{\theta}_{\alpha} \Phi-\epsilon^{\alpha} \theta_{\alpha} \bar{\Phi}\right), & & \\
\delta \Phi & =i \epsilon^{\alpha} W_{\alpha}, & \delta \bar{\Phi} & =i \bar{\epsilon}^{\alpha} \bar{W}_{\alpha}, \\
\delta \mathcal{Q}_{ \pm} & =\mp \frac{1}{4} \bar{\nabla}^{2}\left(\bar{\epsilon}^{\alpha} \bar{\theta}_{\alpha} \overline{\mathcal{Q}}_{\mp}\right), & \delta \overline{\mathcal{Q}}_{ \pm}=\mp \frac{1}{4} \nabla^{2}\left(\epsilon^{\alpha} \theta_{\alpha} \mathcal{Q}_{\mp}\right),
\end{array}
$$

where $\mathcal{Q}_{ \pm}$are as in (3.44). For non-vanishing photon mass, $\mathfrak{m} \neq 0$, the action (4.1) is invariant under (4.2) only for the real supersymmetry parameter $\bar{\epsilon}_{\alpha}=\epsilon_{\alpha}$. This means that for generic $\mathfrak{m}$ the model (4.1) describes the $\mathcal{N}=(3,3)$ supersymmetric electrodynamics while for $\mathfrak{m}=0$ the supersymmetry extends up to $\mathcal{N}=(4,4)$. This scenario is completely analogous to the three-dimensional $\mathcal{N}=4$ electrodynamics which can have only reduced $\mathcal{N}=3$ supersymmetry when the topological Chern-Simons mass term is turned on [41, 42]. In our case, in (4.1) we keep non-vanishing photon mass $\mathfrak{m}$ in order to get rid of IR singularities of Feynman graphs beyond one loop. The one-loop contributions to the effective action, however, are independent of $\mathfrak{m}$ and have the same form for both $\mathcal{N}=(3,3)$ and $\mathcal{N}=(4,4)$ cases.

For quantizing the theory, we perform the background-quantum splitting

$$
V \rightarrow V+e v, \quad \Phi \rightarrow \Phi+e \phi
$$


while the hypermultiplet $\left(\mathcal{Q}_{+}, \mathcal{Q}_{-}\right)$is considered as the 'quantum' superfield which will be integrated out in the path integral. The background gauge superfield $V$ is constrained by (2.34) and (2.35) while $\Phi$ is simply constant

$$
D_{\alpha} \Phi=0, \quad \bar{D}_{\alpha} \bar{\Phi}=0 .
$$

After adding the gauge fixing term (3.45), the 'quantum' fields are described by the action

$$
\begin{aligned}
S_{\text {quant }}= & S_{2}+S_{\text {int }}, \\
S_{2}= & -\int d^{2 \mid 4} z\left[v(\square-H) v+\frac{1}{2} \phi \bar{\phi}+\mathcal{Q}_{+} \overline{\mathcal{Q}}_{+}+\mathcal{Q}_{-} \overline{\mathcal{Q}}_{-}\right] \\
& +\left[\int d^{2 \mid 2} z\left(\frac{i \mathfrak{m}}{4} \phi^{2}-\mathcal{Q}_{+} \Phi \mathcal{Q}_{-}\right)+\text {c.c. }\right] \\
S_{\text {int }}= & -2 \int d^{2 \mid 4} z\left[e\left(\mathcal{Q}_{+} \overline{\mathcal{Q}}_{+}-\mathcal{Q}_{-} \overline{\mathcal{Q}}_{-}\right) v+e^{2}\left(\mathcal{Q}_{+} \overline{\mathcal{Q}}_{+}+\mathcal{Q}_{-} \overline{\mathcal{Q}}_{-}\right) v^{2}\right] \\
& -e \int d^{2 \mid 2} z \mathcal{Q}_{+} \phi \mathcal{Q}_{-}+e \int d^{2 \mid 2} \bar{z} \overline{\mathcal{Q}}_{+} \bar{\phi} \overline{\mathcal{Q}}_{-}+O\left(e^{3}\right) .
\end{aligned}
$$

As compared with (3.46), in (4.5) there are two essential modifications: (i) in (4.5b) we have the background chiral superfield $\Phi$ in place of the mass $m$; (ii) in the last line in $(4.5 \mathrm{c}$ ) there are two additional vertices with the quantum chiral superfield $\phi$ and its conjugate $\bar{\phi}$. Taking these features into account, one can readily generalize the $\mathcal{N}=(2,2)$ effective action (3.52a) to the $\mathcal{N}=(4,4)$ (or, rather, $\mathcal{N}=(3,3)$ ) case

$$
\begin{aligned}
\Gamma & =\Gamma^{(1)}+\Gamma^{(2)}, \\
\Gamma^{(1)} & =i \operatorname{Tr} \ln \left(\square_{+}+\Phi \bar{\Phi}\right), \\
\Gamma^{(2)} & =\Gamma_{\mathrm{A}}+\Gamma_{\mathrm{B}}+\Gamma_{\mathrm{C}}+\Gamma_{\mathrm{D}},
\end{aligned}
$$

where

$$
\begin{aligned}
\Gamma_{\mathrm{A}}= & -2 e^{2} \int d^{2 \mid 4} z d^{2 \mid 4} z^{\prime} G_{+-}\left(z, z^{\prime}\right) G_{+-}\left(z^{\prime}, z\right) G_{v}\left(z, z^{\prime}\right), \\
\Gamma_{\mathrm{B}}= & -2 e^{2} \int d^{2 \mid 4} z d^{2 \mid 4} z^{\prime} \Phi \bar{\Phi} G_{+}\left(z, z^{\prime}\right) G_{-}\left(z, z^{\prime}\right) G_{v}\left(z, z^{\prime}\right), \\
\Gamma_{\mathrm{C}}= & 2 e^{2} \int d^{2 \mid 4} z d^{2 \mid 4} z^{\prime} G_{+-}\left(z, z^{\prime}\right) G_{+-}\left(z, z^{\prime}\right) G_{0}\left(z, z^{\prime}\right), \\
\Gamma_{\mathrm{D}}= & \frac{i e^{2} \mathfrak{m}}{4} \int d^{2 \mid 2} z d^{2 \mid 2} z^{\prime} \Phi^{2} G_{+}\left(z, z^{\prime}\right) G_{+}\left(z^{\prime}, z\right) \bar{D}^{2} G_{0}\left(z, z^{\prime}\right) \\
& +\frac{i e^{2} \mathfrak{m}}{4} \int d^{2 \mid 2} \bar{z} d^{2 \mid 2} \bar{z}^{\prime} \bar{\Phi}^{2} G_{-}\left(z, z^{\prime}\right) G_{-}\left(z^{\prime}, z\right) D^{2} G_{0}\left(z, z^{\prime}\right) .
\end{aligned}
$$

Here $G_{v}\left(z, z^{\prime}\right)$ is given by (3.49) while $G_{0}\left(z, z^{\prime}\right)$ is simply

$$
G_{0}\left(z, z^{\prime}\right)=\frac{1}{\square+\mathfrak{m}^{2}} \delta^{2 \mid 4}\left(z-z^{\prime}\right)=-i \int_{0}^{\infty} d s e^{-i s \mathfrak{m}^{2}} K_{0}\left(z, z^{\prime} \mid s\right) .
$$

Below, we compute separately the one- and two-loop contributions to the effective action (4.6). 


\subsection{One-loop effective action and the Wess-Zumino term}

Recall that we consider the approximation (4.4) which means that we discard any derivatives of (anti)chiral superfield $\Phi(\bar{\Phi})$. In this case the procedure of computation of the one-loop effective action (4.6b) is exactly the same as in section 3.3.2 for the $\mathcal{N}=(2,2)$ SQED. Thus, we can readily generalize the result $(3.53)$ to the case of $\mathcal{N}=(4,4)$ SQED

$$
\Gamma^{(1)}=-\frac{i}{8 \pi} \int d^{2 \mid 2} z \int_{0}^{\infty} d s W^{2} e^{-i s(\Sigma \bar{\Sigma}+\Phi \bar{\Phi})} \frac{\tanh (s f / 2)}{s f / 2}+c . c .
$$

The effective action (4.9) is represented as a functional in (anti)chiral superspace. It is instructive to rewrite it in the full $\mathcal{N}=(2,2)$ superspace. Following the same procedure as in section 3.3.2, we find

$$
\begin{aligned}
\Gamma^{(1)}= & \frac{1}{4 \pi} \int d^{2 \mid 4} z\left[\ln \Sigma \ln \bar{\Sigma}+\operatorname{Li}_{2}\left(-\frac{\Phi \bar{\Phi}}{\Sigma \bar{\Sigma}}\right)\right] \\
& +\frac{i}{4 \pi} \int d^{2 \mid 4} z \int_{0}^{\infty} d s e^{-i s(\Sigma \bar{\Sigma}+\Phi \bar{\Phi})} \frac{W^{2} \bar{W}^{2}}{f^{2}}\left(\frac{\tanh (s f / 2)}{s f / 2}-1\right) .
\end{aligned}
$$

The terms in the first line in (4.10) are leading in the derivative expansion of the effective action while the terms in the second line correspond to higher-derivative corrections. The leading terms

$$
\begin{aligned}
\Gamma_{\text {leading }} & =\int d^{2 \mid 4} z K^{(1)}(\Sigma, \bar{\Sigma} ; \Phi, \bar{\Phi}), \\
K^{(1)}(\Sigma, \bar{\Sigma} ; \Phi, \bar{\Phi}) & =\frac{1}{4 \pi}\left[\ln \Sigma \ln \bar{\Sigma}+\operatorname{Li}_{2}\left(-\frac{\Phi \bar{\Phi}}{\Sigma \bar{\Sigma}}\right)\right]
\end{aligned}
$$

deserve several comments.

First of all, we point out the similarity of the superfield expression (4.11) with the lowenergy effective action of four-dimensional $\mathcal{N}=4$ SYM theory in $\mathcal{N}=2$ superspace which was constructed in [14]. Indeed, (4.11b) contains the term $\ln \Sigma \ln \bar{\Sigma}$ which is analogous to the non-holomorphic potential for $\mathcal{N}=24 \mathrm{~d}$ superfield strength while the other terms are very similar to the hypermultiplet completion of the non-holomorphic potential which was constructed in [14]. Surprisingly, such terms in $2 \mathrm{~d}$ and $4 \mathrm{~d}$ cases are described by the same $\mathrm{Li}_{2}$ function and have very similar form although they are given in very different superspaces and for different models. Recall that the $\mathcal{N}=4$ susy-complete effective action in $4 \mathrm{~d} \mathcal{N}=4 \mathrm{SYM}$ theory was derived originally in [14] by imposing the requirement of invariance under hidden supersymmetry while in subsequent works this effective action was found by direct quantum computations in superspace [43-45] (see also [46] for a review). In our case, we obtained (4.11) as the leading part of the one-loop effective action in $\mathcal{N}=(4,4)$ SQED although originally it was found in [1] as a susy completion of the non-holomorphic potential (1.2).

The mentioned above similarity of (4.11) with the low-energy effective action in $4 \mathrm{~d}$ $\mathcal{N}=4$ SYM theory is even deeper. As was demonstrated in [47, 48] (see also [49] for a review), the structure of leading terms in the low-energy effective action in $\mathcal{N}=4 \mathrm{SYM}$ theory can be recovered from the fact that it contains the Wess-Zumino term for scalar 
fields. This Wess-Zumino term is known to appear in the low-energy theory as a result of 't Hooft anomaly matching for SU(4) R-symmetry of $\mathcal{N}=4$ SYM theory [15]. Surprisingly, the effective action (4.11) may be given exactly the same interpretation. Indeed, in [1] the action of the form (4.11) was proposed as a superfield generalization of a two-dimensional sigma-model with the Wess-Zumino term. In our case, the appearance of this term in the low-energy effective action is well understood. Classically, the $\mathcal{N}=(4,4)$ electrodynamics (4.1) respects the $\mathrm{SU}(2) \times \mathrm{SU}(2)$ symmetry which is the R-symmetry of $\mathcal{N}=(4,4)$ Poincaré superalgebra. However, because of 't Hooft anomaly, this symmetry cannot be realized explicitly in the low-energy theory but is still the symmetry of the effective Lagrangian up to full derivative terms. Recall that the effective action (4.11) is obtained upon integrating out the hypermultiplet $\left(Q_{+}, Q_{-}\right)$which contains chiral fermions with respect to the R-symmetry group. Thus, in the low-energy theory the Wess-Zumino term must appear as a response to the change of the number of chiral fermions since the total contribution to the anomaly should be the same regardless of the energy scale. This is the essence of the 't Hooft anomaly matching argument [11].

Let us derive the Wess-Zumino term for scalar fields from the superfield action (4.11). The scalars appear in the component field expansion of $\Phi$ and $\Sigma$ as follows

$$
\begin{aligned}
& \Phi=\phi+i \theta^{\alpha} \bar{\theta}^{\beta} \gamma_{\alpha \beta}^{m} \partial_{m} \phi+\frac{1}{4} \theta^{2} \bar{\theta}^{2} \square \phi+\ldots, \\
& \Sigma=\sigma-i \theta^{\alpha} \bar{\theta}^{\beta}\left(\gamma_{m}\right)_{\alpha \beta} \varepsilon^{m n} \partial_{n} \sigma-\frac{1}{4} \theta^{2} \bar{\theta}^{2} \square \sigma+\ldots,
\end{aligned}
$$

where dots stand for other component fields. Substituting these expressions into (4.11) and integrating over the Grassmann variables one readily finds in the component field expansion the Wess-Zumino term for the scalar fields

$$
S_{\mathrm{WZ}}=\frac{1}{2 \pi} \int d^{2} x \frac{\phi \bar{\phi}}{\phi \bar{\phi}+\sigma \bar{\sigma}} \varepsilon^{m n} \partial_{m} \alpha \partial_{n} \beta
$$

where $\alpha$ and $\beta$ are phases of the complex scalars $\phi$ and $\sigma$

$$
\phi=|\phi| e^{i \alpha}, \quad \sigma=|\sigma| e^{i \beta} .
$$

The action (4.14) is explicitly invariant under $\mathrm{U}(1) \times \mathrm{U}(1)$ symmetry which shifts the phases $\alpha$ and $\beta$. This symmetry is the subgroup of the full $\mathrm{SU}(2) \times \mathrm{SU}(2) \mathrm{R}$-symmetry group of the theory. It is possible to show that (4.14) is implicitly invariant under $\mathrm{SU}(2) \times \mathrm{SU}(2) \simeq$ $\mathrm{SO}(4)$ since this is the symmetry of the Wess-Zumino term modulo total derivative terms. To show this, let us introduce real scalars $X_{A}=\left(X_{1}, X_{2}, X_{3}, X_{4}\right)$ which transform as a vector under $\mathrm{SO}(4)$

$$
\phi=X_{1}+i X_{2}, \quad \sigma=X_{3}+i X_{4} .
$$

Then, the action (4.14) can be rewritten in the form of integral over a three-dimensional space $\Omega$ which has standard $2 \mathrm{~d}$ Minkowski space as its boundary, $\partial \Omega=\mathbb{R}^{1,1}$ (see e.g. [47] for details)

$$
S_{\mathrm{WZ}}=\frac{1}{6 \pi} \int_{\Omega} d^{3} x \frac{1}{\left(X_{A} X_{A}\right)^{2}} \varepsilon^{A B C D} \varepsilon^{m n p} X_{A} \partial_{m} X_{B} \partial_{n} X_{C} \partial_{p} X_{D}
$$




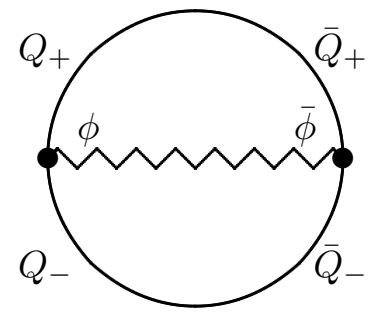

Type C

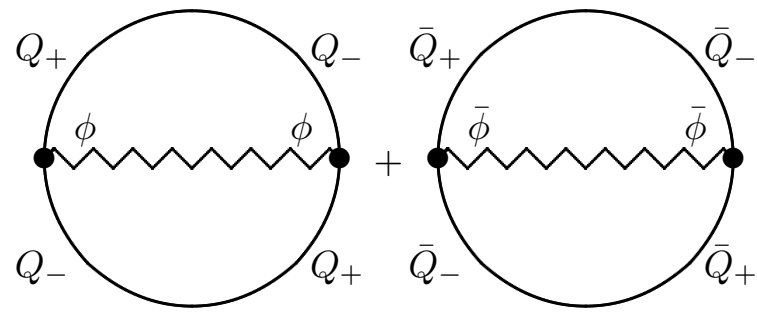

Type D

Figure 2. Two-loop supergraphs in $\mathcal{N}=(4,4)$ SQED which involve propagators $\langle\phi \bar{\phi}\rangle,\langle\phi \phi\rangle$ and $\langle\bar{\phi} \bar{\phi}\rangle$.

where $\varepsilon^{A B C D}$ and $\varepsilon^{m n p}$ are antisymmetric tensors. The Wess-Zumino term in the form (4.17) has explicit $\mathrm{SO}(4)$ symmetry.

One can reverse the arguments: once we know that the Wess-Zumino term (4.17) appears in the low-energy effective action of $\mathcal{N}=(4,4)$ SQED, we can immediately find (4.11) as its supersymmetric generalization. However, performing perturbative quantum computations we uncover not only the leading term (4.11) in the low-energy effective action, but also higher-derivative corrections which are encoded in the second line of (4.10).

We point out once more that (4.14) is explicitly invariant under $\mathrm{U}(1) \times \mathrm{U}(1) \simeq$ $\mathrm{SO}(2) \times \mathrm{SO}(2)$ which is one of the maximal subgroups of the full R-symmetry group $\mathrm{SO}(4)$. However, there are two more inequivalent maximal subgroups: $\mathrm{SO}(3) \simeq \mathrm{SU}(2)$ and $\mathrm{SU}(2) \times \mathrm{U}(1)$. We speculate that these subgroups may be made manifest in other superfield descriptions of the $\mathcal{N}=(4,4)$ gauge theory such as the harmonic superspace [50-54]. Recall that in the $4 \mathrm{~d} \mathcal{N}=4 \mathrm{SYM}$ theory the careful account of all maximal subgroups of the SU(4) R-symmetry group resulted in different but equivalent superfield descriptions of the low-energy effective action $[47-49,55]$. It is tempting to develop similar ideas for the low-energy effective action in $2 \mathrm{~d}$ supersymmetric gauge theories.

\subsection{Vanishing of two-loop corrections to generalized Kähler potential}

In the previous section we computed one-loop effective action which contains the term (4.11) as the leading part in the derivative expansion. In [10] it was claimed that this potential is non-renormalized by higher-loop quantum corrections. This section aims to demonstrate explicitly that two-loop quantum corrections to the generalized Kähler potential (4.11b) cancel among each other.

In the two-loop expansion of effective action (4.6c), the terms $\Gamma_{\mathrm{A}}$ and $\Gamma_{\mathrm{B}}$ can be represented by Feynman graphs which have the same structure as those in the $\mathcal{N}=(2,2)$ SQED given in figure 1 . The terms $\Gamma_{\mathrm{C}}$ and $\Gamma_{\mathrm{D}}$ are new since they involve the propagators for the (anti)chiral superfield $\phi(\bar{\phi})$. These terms are represented by the Feynman graphs in figure 2. To find the contributions to the effective action from these terms it is sufficient to consider the vector multiplet background constrained by (3.62) and (4.4). 
The details of computations of contributions to the effective action (4.7a) and (4.7b) are exactly the same as those in section 3.3.3. We can immediately generalize the results (3.70) and (3.75) to the $\mathcal{N}=(4,4)$ case

$$
\begin{aligned}
\Gamma_{\mathrm{A}} & =\frac{i e^{2}}{8 \pi^{2}} \int d^{2 \mid 4} z \int_{0}^{\infty} \frac{d s d t d u}{t u+s u+s t} e^{-i u \mathfrak{m}^{2}} e^{-i(s+t)(\Phi \bar{\Phi}+\Sigma \bar{\Sigma})}, \\
\Gamma_{\mathrm{B}} & =0 .
\end{aligned}
$$

It is easy to argue that the contribution to the effective action (4.7d) vanishes. Indeed, the propagator $G_{0}$ (4.8) contains the delta-function which implies that we need to consider the heat kernels $K_{+}$and $K_{-}$at coincident points. As follows from (3.20), $K_{+}\left(z, z^{\prime} \mid s\right) \times$ $\left.K_{+}\left(z^{\prime}, z \mid t\right)\right|_{\zeta=0}=0$. Thus,

$$
\Gamma_{\mathrm{D}}=0 .
$$

It remains to consider the contribution $\Gamma_{\mathrm{C}}$ to the effective action. Substituting here the propagators (3.64) and (4.8) we have

$$
\begin{aligned}
\Gamma_{\mathrm{C}} & =2 i e^{2} \int d^{2 \mid 4} z d^{2 \mid 4} z^{\prime} \int_{0}^{\infty} d s d t d u K_{+-}\left(z, z^{\prime} \mid s\right) K_{+-}\left(z, z^{\prime} \mid t\right) K_{0}\left(z, z^{\prime} \mid u\right) e^{-i(s+t) \Phi \bar{\Phi}} e^{-i u \mathfrak{m}^{2}} \\
& =-\frac{2 i e^{2}}{(4 \pi)^{3}} \int d^{2 \mid 4} z d^{2} \rho \int_{0}^{\infty} \frac{d s d t d u}{s t u} e^{-i(s+t)(\Sigma \bar{\Sigma}+\Phi \bar{\Phi})} e^{-i \frac{\rho^{2}}{4}\left(s^{-1}+t^{-1}+u^{-1}\right)} e^{-i u \mathfrak{m}^{2}}
\end{aligned}
$$

After integration over $d^{2} \rho$ it becomes evident that this expression contributes to the effective action with the opposite sign to (4.18)

$$
\Gamma_{\mathrm{C}}=-\frac{i e^{2}}{8 \pi^{2}} \int d^{2 \mid 4} z \int_{0}^{\infty} \frac{d s d t d u}{s t+s u+t u} e^{-i u \mathfrak{m}^{2}} e^{-i(s+t)(\Sigma \bar{\Sigma}+\Phi \bar{\Phi})} .
$$

Thus, we conclude that the sum of the terms (4.18), (4.19), (4.20) and (4.22) vanishes

$$
\Gamma^{(2)}=\Gamma_{\mathrm{A}}+\Gamma_{\mathrm{B}}+\Gamma_{\mathrm{C}}+\Gamma_{\mathrm{D}}=0 .
$$

We stress that this does not mean that the complete two-loop effective action vanishes, but just implies that there are no two-loop quantum corrections to (4.11b).

The non-renormalization of the generalized Kähler potential $K(\Sigma, \bar{\Sigma} ; \Phi, \bar{\Phi})$ in $(4,4)$ gauge theories was claimed in [10]. In this section, we have explicitly demonstrated the absence of two-loop quantum corrections to this potential. There are also purely fieldtheoretical arguments that all higher-loop quantum correction to this function vanish and $K(\Sigma, \bar{\Sigma} ; \Phi, \bar{\Phi})$ is one-loop exact. Indeed, in the previous section it was demonstrated that this potential is responsible for the Wess-Zumino term for scalar fields (4.14). It is wellknown that appearance of Wess-Zumino terms in low-energy effective action is strictly one-loop effect associated with the 't Hooft anomaly matching [11]. The form of the WessZumino term (4.17) as well as the coefficient in front of this action are rigidly fixed by topological arguments (see e.g. [9]). Therefore, the function (4.11b) cannot receive any higher-loop corrections, and we end up with the exact result

$$
K(\Sigma, \bar{\Sigma} ; \Phi, \bar{\Phi})=\frac{1}{2 e^{2}}(\Sigma \bar{\Sigma}-\Phi \bar{\Phi})+\frac{1}{4 \pi}\left[\ln \Sigma \ln \bar{\Sigma}+\operatorname{Li}_{2}\left(-\frac{\Phi \bar{\Phi}}{\Sigma \bar{\Sigma}}\right)\right] .
$$


The corresponding sigma-model metric reads

$$
d s^{2}=d \Sigma d \bar{\Sigma} \partial_{\Sigma} \partial_{\bar{\Sigma}} K-d \Phi d \bar{\Phi} \partial_{\Phi} \partial_{\bar{\Phi}} K=(d \Sigma d \bar{\Sigma}+d \Phi d \bar{\Phi})\left(\frac{1}{2 e^{2}}+\frac{1}{\Sigma \bar{\Sigma}+\Phi \bar{\Phi}}\right) .
$$

As is demonstrated in [4], this sigma-model corresponds to the generalized Kähler geometry with $\mathcal{N}=(4,4)$ extended supersymmetry. This geometry possesses non-trivial torsion which can also be read off from (4.11b). Here we have demonstrated that this generalized Kähler potential naturally arises as the leading term in the low-energy effective action in $\mathcal{N}=(4,4)$ gauge theory on the Coulomb branch.

\section{Conclusions}

In this paper, we have studied two-loop quantum corrections to the low-energy effective actions in the $\mathcal{N}=(2,2)$ and $\mathcal{N}=(4,4)$ SQED. On the Coulomb branch, leading terms in the effective action in $\mathcal{N}=(2,2)$ SQED are represented by a superpotential and a Kähler potential for superfield strengths described by a twisted chiral superfield $\Sigma$. Although, at one-loop order, these potentials were studied long ago [2], to the best of our knowledge the two-loop quantum corrections to the effective Kähler potential (3.80) have not been presented before. The corresponding sigma-model metric in the two-loop approximation is given by (3.83). We point out that this metric depends on the vector multiplet mass $\mathfrak{m}$ and is singular in the limit $\mathfrak{m} \rightarrow 0$. This is a new feature of the two-dimensional case as compared with the low-energy effective action of three-dimensional [23] and four-dimensional [28, 34] SQED where it was well-defined for massless vector multiplet.

In the $\mathcal{N}=(4,4)$ SQED, the leading part of the low-energy effective action is described by the generalized Kähler potential $K(\Sigma, \bar{\Sigma} ; \Phi, \bar{\Phi})$ where $\Phi$ is a chiral and $\Sigma$ is a twisted chiral superfields. We show that this potential is one-loop exact and is given by (4.24). This potential was introduced for the first time in [1] in the study of two-dimensional sigma models with torsion which originates from the Wess-Zumino term for scalar fields. In our case, we demonstrate that the Wess-Zumino term is the integral part of the low-energy effective action associated with the 't Hooft anomaly matching for the $\mathrm{SU}(2) \times \mathrm{SU}(2) \mathrm{R}$ symmetry. The from of the potential $K$ appears surprisingly similar to the low-energy effective action in $4 \mathrm{~d} \mathcal{N}=4$ SYM theory obtained in [14].

We have studied two-loop effective action in two-dimensional SQED on the Coulomb branch. In is also very interesting to investigate the structure of the effective action on the Higgs branch. Some of the leading terms in this effective action were discussed in the recent work [56] but the form of higher-loop quantum correction is unknown. Another interesting problem is the study of quantum aspects of two-dimensional gauge theories with semichiral multiplets $[8,36,37]$. Only some limited results in this direction are available [57], but the structure of quantum corrections to the generalized Kähler potential remains unknown.

\section{Acknowledgments}

I am very grateful to E.A. Ivanov for useful discussions. I acknowledge the support from the RFBR grant No 15-02-06670. 


\section{A $\mathcal{N}=(2,2)$ superspace conventions}

In this paper, we use the two-dimensional $\mathcal{N}=(2,2)$ superspace which appears by the dimensional reduction from the three-dimensional $\mathcal{N}=2$ superspace or from the fourdimensional standard $\mathcal{N}=1$ superspace. Therefore, we employ the superfield notation and conventions which are very close to the ones used in the series of papers [23-27] devoted to the study of the low-energy effective actions in three-dimensional superfield theories.

The $2 \mathrm{~d} \mathcal{N}=(2,2)$ superspace is parametrized by the coordinates $z^{A}=\left(x^{m}, \theta^{\alpha}, \bar{\theta}_{\alpha}\right)$, where $x^{m}=\left(x^{0}, x^{1}\right)$ are the Minkowski space coordinates and $\theta^{\alpha}=\left(\theta^{1}, \theta^{2}\right)$ are Grassmann coordinates $\left(\bar{\theta}_{\alpha}=\left(\theta_{\alpha}\right)^{*}\right.$ are their complex conjugate). The spinor indices are raised and lowered by means of the antisymmetric $\varepsilon$-tensor, $\theta^{\alpha}=\varepsilon^{\alpha \beta} \theta_{\beta}, \theta_{\alpha}=\varepsilon_{\alpha \beta} \theta^{\beta}, \varepsilon_{12}=\varepsilon^{21}=1$.

One of the possible choices for the two-dimensional gamma-matrices $\left(\gamma^{m}\right)=\left(\gamma^{m}\right)_{\alpha}^{\beta}$ may be $\gamma^{0}=-i \sigma_{2}, \gamma^{1}=\sigma_{1}$, where $\sigma_{i}$ are the Pauli matrices. The gamma-matrices obey the Clifford algebra

$$
\left\{\gamma^{m}, \gamma^{n}\right\}=-2 \eta^{m n} \mathbf{1}_{2 \times 2}, \quad \eta^{m n}=\operatorname{diag}(1,-1),
$$

and possess the following orthogonality and completeness relations

$$
\left(\gamma^{m}\right)_{\alpha}^{\beta}\left(\gamma^{n}\right)_{\beta}^{\alpha}=-2 \eta^{m n}, \quad\left(\gamma^{m}\right)_{\alpha}^{\beta}\left(\gamma_{m}\right)_{\sigma}^{\rho}=\varepsilon^{\beta \rho} \varepsilon_{\sigma \alpha}-\delta_{\alpha}^{\rho} \delta_{\sigma}^{\beta}+\left(\gamma^{3}\right)_{\alpha}^{\beta}\left(\gamma^{3}\right)_{\sigma}^{\rho},
$$

where $\gamma^{3}=\gamma^{1} \gamma^{0}$ is the chirality matrix. The chiral projectors are $P_{ \pm}=\frac{1}{2}\left(\mathbf{1} \pm \gamma^{3}\right)$.

The covariant spinor derivatives may be chosen in the form

$$
D_{\alpha}=\frac{\partial}{\partial \theta^{\alpha}}+i \bar{\theta}^{\beta}\left(\gamma^{m}\right)_{\alpha \beta} \partial_{m}, \quad \bar{D}_{\alpha}=-\frac{\partial}{\partial \bar{\theta}^{\alpha}}-i \theta^{\beta}\left(\gamma^{m}\right)_{\alpha \beta} \partial_{m},
$$

with the following anti-commutation relation

$$
\left\{D_{\alpha}, \bar{D}_{\beta}\right\}=-2 i\left(\gamma^{m}\right)_{\alpha \beta} \partial_{m} .
$$

The integration measure in the full $\mathcal{N}=(2,2)$ superspace is defined as

$$
d^{2 \mid 4} z \equiv d^{2} x d^{4} \theta=\frac{1}{16} d^{2} x D^{2} \bar{D}^{2}, \quad \text { so that } \quad \int d^{2} x f(x)=\int d^{2 \mid 4} z \theta^{2} \bar{\theta}^{2} f(x),
$$

for some field $f(x)$. Here we adopt the following conventions for contractions of spinor indices

$$
D^{2}=D^{\alpha} D_{\alpha}, \quad \bar{D}^{2}=\bar{D}^{\alpha} \bar{D}_{\alpha}, \quad \theta^{2}=\theta^{\alpha} \theta_{\alpha}, \quad \bar{\theta}^{2}=\bar{\theta}^{\alpha} \bar{\theta}_{\alpha} .
$$

The chiral and antichiral subspaces are parametrized by the coordinates $z_{+}=\left(x_{+}^{m}, \theta_{\alpha}\right)$ and $z_{-}=\left(x_{-}^{m}, \bar{\theta}_{\alpha}\right)$, correspondingly, where

$$
x_{ \pm}^{m}=x^{m} \pm i \gamma_{\alpha \beta}^{m} \theta^{\alpha} \bar{\theta}^{\beta} .
$$

The integration measure in the chiral subspace $d^{2 \mid 2} z \equiv d^{2} x d^{2} \theta$ is related to the full superspace measure (A.5) as

$$
d^{2 \mid 4} z=-\frac{1}{4} d^{2 \mid 2} z \bar{D}^{2}=-\frac{1}{4} d^{2 \mid 2} \bar{z} D^{2}, \quad \text { so that } \quad \int d^{2} x f(x)=\int d^{2 \mid 2} z \theta^{2} f\left(x_{+}\right) .
$$


Given a two-component spinor $\psi_{\alpha}$, we can consider each of its components independently as they are Lorentz-covariant and appear as the $P_{ \pm}$projections of $\psi$,

$$
\psi_{\alpha} \equiv\left(\psi_{+}, \psi_{-}\right), \quad \psi_{ \pm}=P_{ \pm} \psi
$$

In a similar way, for a spinor with the upper spinor index, $\chi^{\alpha}$, we have $\chi^{\alpha} \equiv\left(\chi^{+}, \chi^{-}\right)=$ $\left(-\chi_{-}, \chi_{+}\right)$. As a consequence, it is possible to introduce twisted chiral coordinates $\tilde{z}_{+}=$ $\left(\tilde{x}_{+}^{m}, \theta_{+}, \bar{\theta}_{-}\right)$and the twisted antichiral ones $\tilde{z}_{-}=\left(\tilde{x}_{-}^{m}, \theta_{-}, \bar{\theta}_{+}\right)$, where

$$
\tilde{x}_{ \pm}^{m}=x^{m} \pm i\left(\gamma^{m}\right)_{\alpha}^{\beta} \gamma_{\beta \gamma}^{3} \theta^{\alpha} \bar{\theta}^{\gamma}
$$

The integration measures over these subspaces are denoted by $d^{2 \mid 2} \tilde{z}$ and $d^{2 \mid 2} \tilde{\bar{z}}$. They are related to the full superspace measure (A.5) as

$$
d^{2 \mid 4} z=d^{2 \mid 2} \tilde{z} \frac{1}{2} D_{+} \bar{D}_{-}=d^{2 \mid 2} \tilde{z} \frac{1}{2} \bar{D}_{+} D_{-} .
$$

The existence of twisted chiral subspace in addition to the conventional chiral one is the crucial feature of the two-dimensional superspace as compared with the higherdimensional story.

Open Access. This article is distributed under the terms of the Creative Commons Attribution License (CC-BY 4.0), which permits any use, distribution and reproduction in any medium, provided the original author(s) and source are credited.

\section{References}

[1] M. Roček, K. Schoutens and A. Sevrin, Off-shell WZW models in extended superspace, Phys. Lett. B 265 (1991) 303 [INSPIRE].

[2] A. D'Adda, A.C. Davis, P. Di Vecchia and P. Salomonson, An effective action for the supersymmetric $C P^{(N-1)}$ model, Nucl. Phys. B 222 (1983) 45 [InSPIRE].

[3] E. Witten, Phases of $N=2$ theories in two-dimensions, Nucl. Phys. B 403 (1993) 159 [hep-th/9301042] [INSPIRE].

[4] S.J. Gates, Jr., C.M. Hull and M. Roček, Twisted multiplets and new supersymmetric nonlinear $\sigma$-models, Nucl. Phys. B 248 (1984) 157 [INSPIRE].

[5] K. Hori and D. Tong, Aspects of non-abelian gauge dynamics in two-dimensional $N=(2,2)$ theories, JHEP 05 (2007) 079 [hep-th/0609032] [INSPIRE].

[6] E.A. Ivanov and S.O. Krivonos, $N=4$ super-Liouville equation (in Russian), J. Phys. A 17 (1984) L671 [INSPIRE].

[7] E.A. Ivanov, S.O. Krivonos and V.M. Leviant, A new class of superconformal $\sigma$ models with the Wess-Zumino action, Nucl. Phys. B 304 (1988) 601 [INSPIRE].

[8] T. Buscher, U. Lindström and M. Roček, New supersymmetric $\sigma$ models with Wess-Zumino terms, Phys. Lett. B 202 (1988) 94 [InSPIRE].

[9] E. Braaten, T.L. Curtright and C.K. Zachos, Torsion and geometrostasis in nonlinear $\sigma$-models, Nucl. Phys. B 260 (1985) 630 [inSPIRE].

[10] D.-E. Diaconescu and N. Seiberg, The Coulomb branch of $(4,4)$ supersymmetric field theories in two-dimensions, JHEP 07 (1997) 001 [hep-th/9707158] [INSPIRE]. 
[11] G. 't Hooft, Naturalness, chiral symmetry, and spontaneous chiral symmetry breaking, NATO Sci. Ser. B 59 (1980) 135.

[12] N. Seiberg, Supersymmetry and nonperturbative $\beta$-functions, Phys. Lett. B 206 (1988) 75 [INSPIRE].

[13] M. Dine and N. Seiberg, Comments on higher derivative operators in some SUSY field theories, Phys. Lett. B 409 (1997) 239 [hep-th/9705057] [INSPIRE].

[14] I.L. Buchbinder and E.A. Ivanov, Complete $N=4$ structure of low-energy effective action in $N=4$ super Yang-Mills theories, Phys. Lett. B 524 (2002) 208 [hep-th/0111062] [INSPIRE].

[15] K.A. Intriligator, Anomaly matching and a Hopf-Wess-Zumino term in $6 d, N=(2,0)$ field theories, Nucl. Phys. B 581 (2000) 257 [hep-th/0001205] [INSPIRE].

[16] I.L. Buchbinder, Divergences of effective action in external supergauge field (in Russian), Yad. Fiz. 36 (1982) 509 [INSPIRE].

[17] T. Ohrndorf, An example of an explicitly calculable supersymmetric low-energy effective lagrangian: the Heisenberg-Euler lagrangian of supersymmetric QED, Nucl. Phys. B 273 (1986) 165 [INSPIRE].

[18] S.M. Kuzenko and I.N. McArthur, On the background field method beyond one loop: A Manifestly covariant derivative expansion in super Yang-Mills theories, JHEP 05 (2003) 015 [hep-th/0302205] [INSPIRE].

[19] I.N. McArthur, Super-b 4 coefficients, Phys. Lett. B 128 (1983) 194.

[20] I.N. McArthur, Super b4 coefficients in supergravity, Class. Quant. Grav. 1 (1984) 245 [INSPIRE].

[21] I.L. Buchbinder and S.M. Kuzenko, On the structure of the green function of chiral superfield in the supergravity external field (in Russian), Yad. Fiz. 41 (1985) 1671 [INSPIRE].

[22] I.L. Buchbinder and S.M. Kuzenko, Matter superfields in external supergravity: green functions, effective action and superconformal anomalies, Nucl. Phys. B 274 (1986) 653 [INSPIRE].

[23] I.L. Buchbinder, B.S. Merzlikin and I.B. Samsonov, Two-loop low-energy effective actions in $N=2$ and $N=4$ three-dimensional SQED, JHEP 07 (2013) 012 [arXiv:1305.4815] [INSPIRE].

[24] I.L. Buchbinder, N.G. Pletnev and I.B. Samsonov, Effective action of three-dimensional extended supersymmetric matter on gauge superfield background, JHEP 04 (2010) 124 [arXiv: 1003.4806] [INSPIRE].

[25] I.L. Buchbinder, N.G. Pletnev and I.B. Samsonov, Low-energy effective actions in three-dimensional extended SYM theories, JHEP 01 (2011) 121 [arXiv:1010.4967] [INSPIRE].

[26] I.L. Buchbinder, B.S. Merzlikin and I.B. Samsonov, Two-loop effective potentials in general $N=2, D=3$ chiral superfield model, Nucl. Phys. B 860 (2012) 87 [arXiv:1201.5579] [INSPIRE].

[27] I.L. Buchbinder, B.S. Merzlikin and I.B. Samsonov, Two-loop low-energy effective action in Abelian supersymmetric Chern-Simons matter models, Nucl. Phys. B 881 (2014) 42 [arXiv: 1311.5001] [INSPIRE].

[28] S.M. Kuzenko and I.N. McArthur, Low-energy dynamics in $N=2$ super QED: two loop approximation, JHEP 10 (2003) 029 [hep-th/0308136] [INSPIRE]. 
[29] S.M. Kuzenko and I.N. McArthur, On the two loop four derivative quantum corrections in $4 D N=2$ superconformal field theories, Nucl. Phys. B 683 (2004) 3 [hep-th/0310025] [INSPIRE].

[30] S.M. Kuzenko and I.N. McArthur, Relaxed superselfduality and effective action, Phys. Lett. B 591 (2004) 304 [hep-th/0403082] [INSPIRE].

[31] S.M. Kuzenko and I.N. McArthur, Relaxed superselfduality and $N=4$ SYM at two loops, Nucl. Phys. B 697 (2004) 89 [hep-th/0403240] [InSPIRE].

[32] S.M. Kuzenko, Exact propagators in harmonic superspace, Phys. Lett. B 600 (2004) 163 [hep-th/0407242] [INSPIRE].

[33] S.M. Kuzenko, Self-dual effective action of $N=4$ SYM revisited, JHEP 03 (2005) 008 [hep-th/0410128] [INSPIRE].

[34] S.M. Kuzenko and S.J. Tyler, Supersymmetric Euler-Heisenberg effective action: two-loop results, JHEP 05 (2007) 081 [hep-th/0703269] [INSPIRE].

[35] S.R. Coleman, More about the massive Schwinger model, Annals Phys. 101 (1976) 239 [INSPIRE].

[36] U. Lindström, M. Roček, I. Ryb, R. von Unge and M. Zabzine, New $N=(2,2)$ vector multiplets, JHEP 08 (2007) 008 [arXiv:0705.3201] [INSPIRE].

[37] S.J. Gates Jr. and W. Merrell, $D=2 N=(2,2)$ semi chiral vector multiplet, JHEP 10 (2007) 035 [arXiv:0705.3207] [INSPIRE].

[38] K.-i. Shizuya and Y. Yasui, Construction of effective actions in superspace, Phys. Rev. D 29 (1984) 1160 [InSPIRE].

[39] I.L. Buchbinder, N.G. Pletnev and I.B. Samsonov, Background field formalism and construction of effective action for $N=2, D=3$ supersymmetric gauge theories, Phys. Part. Nucl. 44 (2013) 234 [arXiv:1206.5711] [INSPIRE].

[40] I. Huet, D.G.C. McKeon and C. Schubert, Euler-Heisenberg lagrangians and asymptotic analysis in $1+1$ QED, part 1: two-loop, JHEP 12 (2010) 036 [arXiv:1010.5315] [INSPIRE].

[41] H.-C. Kao and K.-M. Lee, Selfdual Chern-Simons systems with an $N=3$ extended supersymmetry, Phys. Rev. D 46 (1992) 4691 [hep-th/9205115] [INSPIRE].

[42] H.-C. Kao, Selfdual Yang-Mills Chern-Simons Higgs systems with an $N=3$ extended supersymmetry, Phys. Rev. D 50 (1994) 2881 [INSPIRE].

[43] I.L. Buchbinder, E.A. Ivanov and A. Yu. Petrov, Complete low-energy effective action in $N=4$ SYM: a direct $N=2$ supergraph calculation, Nucl. Phys. B 653 (2003) 64 [hep-th/0210241] [INSPIRE].

[44] A.T. Banin, I.L. Buchbinder and N.G. Pletnev, One loop effective action for $N=4 S Y M$ theory in the hypermultiplet sector: leading low-energy approximation and beyond, Phys. Rev. D 68 (2003) 065024 [hep-th/0304046] [INSPIRE].

[45] I.L. Buchbinder and N.G. Pletnev, Construction of one-loop $N=4$ SYM effective action on the mixed branch in the harmonic superspace approach, JHEP 09 (2005) 073 [hep-th/0504216] [INSPIRE].

[46] I.L. Buchbinder, E.A. Ivanov and N.G. Pletnev, Superfield approach to the construction of effective action in quantum field theory with extended supersymmetry, Phys. Part. Nucl. $\mathbf{4 7}$ (2016) 291 [INSPIRE].

[47] D.V. Belyaev and I.B. Samsonov, Wess-Zumino term in the $N=4$ SYM effective action revisited, JHEP 04 (2011) 112 [arXiv:1103.5070] [INSPIRE]. 
[48] D.V. Belyaev and I.B. Samsonov, Bi-harmonic superspace for $N=4 D=4$ super Yang-Mills, JHEP 09 (2011) 056 [arXiv:1106.0611] [InSPIRE].

[49] I.L. Buchbinder, E.A. Ivanov and I.B. Samsonov, The low-energy $N=4 S Y M$ effective action in diverse harmonic superspaces, Phys. Part. Nucl. 48 (2017) 333 [arXiv: 1603. 02768] [INSPIRE].

[50] E. Ivanov and A. Sutulin, $\sigma$-models in $(4,4)$ harmonic superspace, Nucl. Phys. B 432 (1994) 246 [Erratum ibid. B 483 (1997) 531] [hep-th/9404098] [INSPIRE].

[51] E.A. Ivanov, On the harmonic superspace geometry of $(4,4)$ supersymmetric $\sigma$-models with torsion, Phys. Rev. D 53 (1996) 2201 [hep-th/9502073] [INSPIRE].

[52] E.A. Ivanov, Off-shell $(4,4)$ supersymmetric $\sigma$-models with torsion as gauge theories in harmonic superspace, Phys. Lett. B 356 (1995) 239 [hep-th/9504070] [INSPIRE].

[53] S. Bellucci and E. Ivanov, $N=(4,4), 2 D$ supergravity in $\mathrm{SU}(2) \times \mathrm{SU}(2)$ harmonic superspace, Nucl. Phys. B 587 (2000) 445 [hep-th/0003154] [INSPIRE].

[54] E. Ivanov and A. Sutulin, Diversity of off-shell twisted $(4,4)$ multiplets in $\mathrm{SU}(2) \times \mathrm{SU}(2)$ harmonic superspace, Phys. Rev. D 70 (2004) 045022 [hep-th/0403130] [INSPIRE].

[55] I.L. Buchbinder, E.A. Ivanov, I.B. Samsonov and B.M. Zupnik, Superconformal $N=3$ SYM low-energy effective action, JHEP 01 (2012) 001 [arXiv:1111.4145] [INSPIRE].

[56] O. Aharony, S.S. Razamat, N. Seiberg and B. Willett, The long flow to freedom, JHEP 02 (2017) 056 [arXiv: 1611.02763] [INSPIRE].

[57] J. Nian and X. Zhang, Dynamics of two-dimensional $\mathcal{N}=(2,2)$ theories with semichiral superfields I, JHEP 11 (2015) 047 [arXiv: 1411.4694] [INSPIRE]. 